Existence of Dynamics for a $1-d$ NLS EQUATION PERTURBed With A GENERALIZED POINT DEFECT

Riccardo Adami and Diego Noja

QUADERNO N. 6/2009 (mp-arc 09-60)

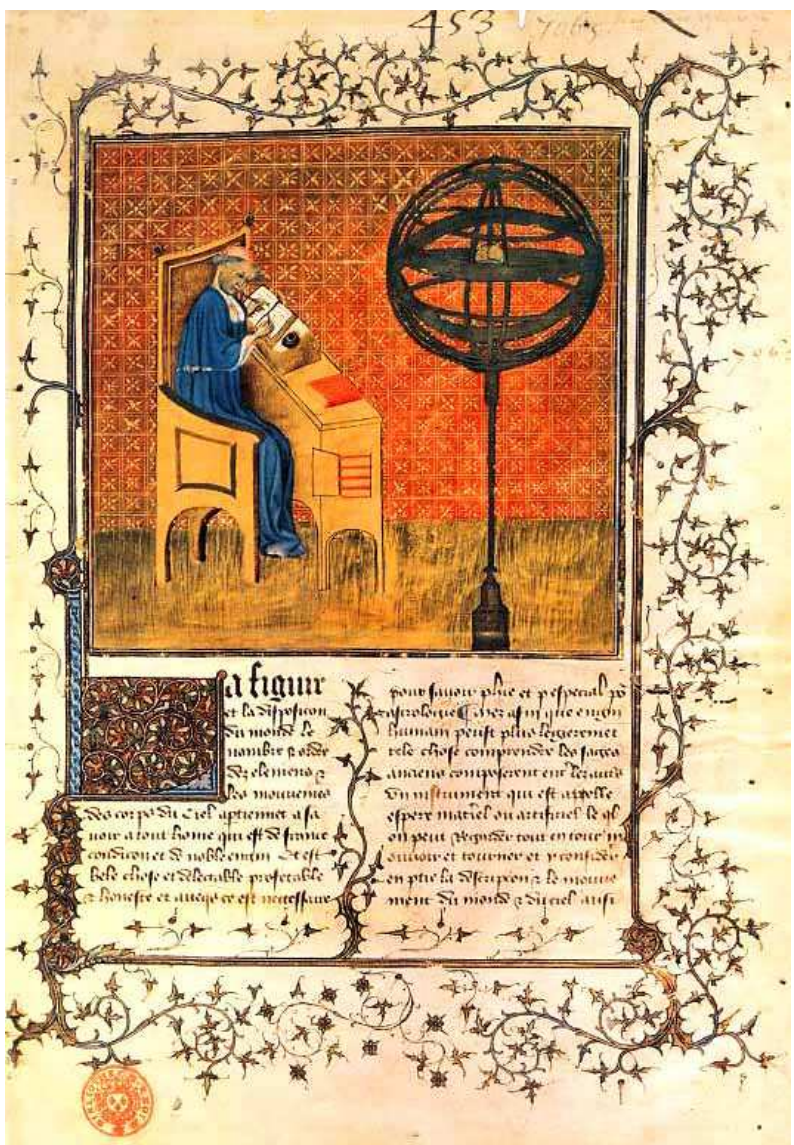


STAMPATO NEL MESE Di APRILE 2009

PRESSO Il Dipartimento di Matematica e ApplicAzioni,

Università degli Studi di Milano-Bicocca, via R. Cozzi 53, 20125 Milano, italia.

DiSPONIBILE IN FORMATO ELETTRONICO SUL SITO www matapp.unimib.it.

Segreteria di Redazione: Ada Osmetti - Giuseppina Cogliandro

tel.: +39026448 5755-5758 fax: +390264485705

Esemplare fuori commercio per il deposito legale agli effetti della Legge 15 aprile 2004 n.106. 


\title{
Existence of dynamics for a 1-d NLS equation perturbed with a generalized point defect
}

\author{
Riccardo Adami and Diego Noja \\ Dipartimento di Matematica e Applicazioni, Università di Milano Bicocca \\ via R. Cozzi, 53, 20125 Milano, Italy
}

April 8, 2009

\begin{abstract}
In the present paper we study the well-posedness for the one-dimensional cubic NLS perturbed by a generic point interaction. Point interactions are described as the 4-parameter family of selfadjoint extensions of the symmetric 1-d laplacian defined on the regular functions vanishing at a point, and in the present context can be interpreted as localized defects interacting with the NLS field. A previously treated special case is given by a NLS equation with a $\delta$ defect (see [21]) which we generalize and extend, as far as well-posedness is concerned, to the whole family of point interactions. We prove existence and uniqueness of the local Cauchy problem in strong form (initial data and evolution in the operator domain of point interactions) and weak form (initial data and evolution in the form domain of point interactions). Correspondingly, a related blowup-eternity alternative is given and studied. Conservation laws of mass and energy are proved for finite energy weak solutions of the problem, which imply global existence of the dynamics. A technical difficulty arises due to the fact that a power nonlinearity does not preserve the form domain for a subclass of point interactions; to overcome it, a technique based on the extension of resolvents of the linear part of the generator to maps between a suitable Hilbert space and the energy space is devised and estimates are given which show the needed regularization properties of the nonlinear flow.
\end{abstract}

\section{Introduction}

The present paper is devoted to the well-posedness of a nonlinear Schrödinger equation with a point defect in dimension one. The Schrödinger equation bears a cubical nonlinearity, and the defect is described by the general point interaction in dimension one. To be precise, the equation to be studied is given by

$$
\left\{\begin{array}{ccc}
i \partial_{t} \psi(t) & = & H \psi(t)+\lambda|\psi(t)|^{2} \psi(t) \\
\psi(0) & = & \psi_{0}
\end{array}\right.
$$

or in weak form

$$
\psi(t)=e^{-i H t} \psi_{0}-i \lambda \int_{0}^{t} d s e^{-i H(t-s)}|\psi(s)|^{2} \psi(s)
$$

where $\psi_{0}$ represents the initial data and $H$ is the Hamiltonian operator generating a point interaction at the origin. Point interactions are singular perturbations of the Laplace operator; restricting the 
Laplacian on the set of regular functions vanishing at a point gives a symmetric non self-adjoint operator; its self-adjoint extensions are by definition point interactions ([5]). In one dimension they form a four-parameter family of s.a. operators ([4]), and they describe in an effective way a variety of situations relevant to the dynamics and scattering of quantum particles acted on by strongly concentrated potentials. In dimension one their domains are characterized by suitable boundary conditions, which apart from the standard ones (i.e. Dirichlet, Neumann, Robin, one sided or two sided), comprise analogous boundary conditions involving the jump of the functions $([\psi])$ or their derivatives at the point where the interaction takes place. Among the main non trivial examples are the well-known $\delta$ interaction (where the boundary condition is $\left[\psi^{\prime}\right](0)=\alpha \psi(0)$ ) often called Fermi pseudopotential in the physics literature, or the $\delta^{\prime}$ interaction $\left([f](0)=\alpha f^{\prime}(0)\right)$. Precise definitions will be given in the next section of the paper. In the case of NLS with $\delta$ interaction there exists a certain amount of literature, physical, numerical and mathematical, concerning the existence of stationary states $([10,27,8,9])$, the asymptotic behaviour in time $([20,21])$, and the reduced dynamics on the stable soliton manifold $([14,18])$. Little is known for the $\delta^{\prime}$ interaction, and nothing in the generic case. Quite generically, equation 1.1 is a prototype of the interactions of nonlinear waves propagating in media in which inhomogeneities are present. A first possible physical interpretation of the model described by the equation 1.1 is given by the interaction of a 1-d Bose condensate with an impurity. To the right and to the left of the point perturbation the Bose condensate satisfies, as an effective equation in the limit of infinite bosons (see for the one dimensional case [1], for the three-dimensional setting [16], [17], [23]), the NLS equation, usually called Gross-Pitaevskii equation in this context. At the defect or impurity location a boundary condition establishes the nature of interaction, and gives the link between the two sides of the condensate. Bose condensates are quantum many-body systems which display typical macroscopic behaviour, measured in an effective way through the scattering length of the underlying two-body interaction. So the use of a point interaction in 1.1 is legitimate if the scale length at which takes place the interaction is far smaller than the characteristic scattering length of the Bose condensate. A second interpretation is of classical origin. It is well-known that the propagation of an optical wave pulse in a nonlinear dispersive medium (such as an optical fiber) gives rise to a nonlinear Schrödinger equation for the evolution of the pulse envelope. The presence of defects or junctions in the fiber can be modeled through boundary conditions, and in the simple and generally adopted case of 1-dimensional propagation along the fiber this corresponds to consider a point perturbation. A different occurrence of an effective point $(\delta)$ interactions is in the study of bimodal optical fibers; these devices are described by two coupled NLS which admit two-soliton solutions; in a typical situation the solitons are one narrow and one wider. At a formal level it turns out that in a suitable limit the pulse propagation is described by a single NLS and the effect of the narrower soliton can be represented by a $\delta$ interaction, at least as far as its influence on the dynamics of the wider one is concerned (see [10] and references therein). The previous applications acquire an additional interest due to the evidence, both on the numerical and rigorous side, of a certain persistence of the soliton behaviour even in the presence of a breaking of translational invariance due to a $\delta$ interaction $([20,21])$; in particular a fast soliton breaks in two pieces, one reflected and one transmitted, the relative amplitude of which being controlled by the scattering matrix of the $\delta$ interaction at least within times long with respect to the interaction time. This is a meaningful phenomenon quite different from orbital stability and indicates a peculiar robustness of the soliton solutions of NLS, even in the case of strong interaction with external perturbation.

Among the possible future perspectives, let us cite the analysis of the dynamics of the system investigated in [12], where the ground states of stationary Maxwell-Schr̈odinger system in a bounded 
domain with a point defect are studied. In such a case the nonlinearity arises from a Hartree type interaction, and is milder than in the usual NLS; nevertheless the results and the techniques are interesting because they apply in dimension three.

As a final quotation, we mention the paper [24], which deals with well-posedness, direct and inverse scattering for a family of NLS with potential terms.

We are not concerned here with a rigorous justification (which is lacking) of the point interaction as an effective model of scatterer or junction in the NLS propagation phenomenon. We assume it as a plausible one and we proceed to show existence and uniqueness in the small and in the large and qualitative properties as energy conservation for the whole family of point interactions. At a rigorous level, well-posedness of the problem 1.2 is well-known for the case of $\delta$ interaction only (see $[21,18])$.

In the following we give a brief summary of the results obtained in the paper and the techniques involved in the proofs. In section 2 the definition and main properties of general point interactions are given. In particular the form domain and quadratic form of the family is completely described (as far as we know this material is not published elsewhere). Moreover, the dual space of form domains are given, in that they are needed in the proof of of well-posedness in energy spaces. In section 3, some estimates of the Gagliardo-Nirenberg type are given for functions in the energy spaces of point interactions. These are needed in the subsequent analysis as a technical tool.

In section 4 we state and prove local existence, uniqueness and blow-up alternative for strong solution to 1.1 (theorem 4.1). By strong solutions we mean solutions $t \rightarrow \psi(t)$ with values in the domain of $H$. Then we prove energy conservation for the strong flow, proposition 4.3, as a consequence of which blow-up does not occur and maximal solutions are in fact global in time. The proof of theorem 4.1 is through a contraction in a suitable neighbourhood of the initial point in the space $\mathcal{X}=C([0, T], D(H)) \cap C^{1}\left([0, T], L^{2}\left(\mathbb{R}^{3}\right)\right)$; the main technical points are a bound on the $L^{\infty}$ norm on $\psi$ and its graph norm $\left\|\left(H_{U}+m\right) \psi\right\|_{L^{2}(\mathbb{R})}$, and an integration by parts in the integral form of problem 1.1 to get a regularization of singular terms due to nonlinearity. The last problem treated is local and global well-posedness in the finite energy space, i.e. form domains. Quite general results of local existence in which the linear part of the generator is self-adjoint are known in the literature (see theorem 3.9.9 in [11] and section 3.7). In such a case one obtains a local existence of a solution $\psi \in C([0, T], X) \cap C^{1}\left([0, T], X^{\star}\right)$, where $X$ is the form domain of the linear part of the generator, if uniqueness is known and a set of hypotheses are satisfied. Here we prefer to proceed in a direct way, because the verification of the hypotheses of the quoted general results for a part of the family of point interactions is not simpler than a direct proof; moreover, we prefer to directly treat the delicate case in which a boundary condition is present in the definition of the energy space: this is indeed the case for a subclass of the family of point interactions, whose form domain is given by $Q_{\omega a}=\left\{\psi \in H^{1}\left(\mathbb{R}^{+}\right) \oplus H^{1}\left(\mathbb{R}^{-}\right)\left|\psi\left(0_{+}\right)=\omega a \psi\left(0_{-}\right),\right| \omega \mid=1, a \in \mathbb{R} \backslash\{0, \pm 1\}\right\}$. In the first place the nonlinearity does not preserve the boundary condition. As a consequence, for these interactions there appear terms in the Duhamel formula which are not in the energy space. To get the relevant estimates, we prove that the resolvent of the linear part can be continuously extended to a suitable Banach space, larger than the dual of the energy spaces, with values still in the energy space. In the end, one obtains for NLS with arbitrary point interactions local and global well-posedness on the form domain of point interaction itself, stated and proved in theorem 6.9.

Our results extend to dynamics on graphs, which is the subject of a paper in preparation.

\subsection{Notation}

Here we fix some basic notation that we will use through the paper. 
1. The symbol $(\psi, \phi)$ denotes the scalar product in $L^{2}(\mathbb{R})$ between the functions $\psi$ and $\phi$, according to the definition

$$
(\psi, \phi)=\int_{\mathbb{R}} \overline{\psi(x)} \phi(x) d x .
$$

2. The symbol $\langle f, \psi\rangle_{X^{\star}, X}$ denotes the duality product between the functional $f \in X^{\star}$ and the vector $\psi \in X$. The space $X$ will be always specified.

3. We denote by $\widehat{\psi}$ or $\mathcal{F} \psi$ the Fourier transform of the function $\psi \in L^{2}(\mathbb{R})$; the convention on the normalization is the one given (when meaningful) by

$$
\widehat{\psi}(k)=(2 \pi)^{-1 / 2} \int_{\mathbb{R}} \psi(x) e^{-i k x} d x .
$$

The symbol $\mathcal{F}$ will be used also to denote the Fourier transform in the distribution space.

4. The symbols $\chi_{ \pm}$denote the characteristic function of the sets $\mathbb{R}^{ \pm}$.

5. Some particular functions will play an important role and we will need to use them frequently. Therefore, we define a notation for them:

$$
\begin{aligned}
\varphi_{ \pm}(x) & =\chi_{ \pm}(x) e^{\mp x} \\
\varphi_{\nu}(x) & =\nu \varphi_{+}(x)+\varphi_{-}(x)
\end{aligned}
$$

Notice that, as a particular case, $\varphi_{1}=e^{-|\cdot|}$. We will use also

$$
\begin{aligned}
\varphi_{ \pm}^{z}(x) & =\chi_{ \pm}(x) e^{\mp \sqrt{z} x} \\
\varphi^{z}(x) & =\varphi_{+}^{z}(x)+\varphi_{-}^{z}(x)=e^{-\sqrt{z}|x|}
\end{aligned}
$$

where $z \in \mathbb{C}, \operatorname{Re} \sqrt{z}>0$

6. The norm of $\psi$ in the space $L^{p}(\mathbb{R})$ is denoted by $\|\psi\|_{p}$, except for $p=2$, in which case we omit the subscript. For any other space we explicitly refer to the space in the subscript (e.g., for the $H^{1}$ norm we use $\left.\|\cdot\|_{H^{1}(\mathbb{R})}\right)$.

\section{Spaces}

We start recalling definitions and basic notions on one-dimensional point interactions. We construct the form domain for the entire family of Hamiltonians of point interaction.

\subsection{Point interactions in dimension one: operators and forms}

By definition, the family of hamiltonian operators describing the dynamics of a particle in dimension one under the influence of a scattering centre located at the origin, is obtained as the set of self-adjoint extensions (s.a.e.) of the operator

$$
\widehat{H}_{0}=-\partial_{x}^{2}
$$

defined on the domain

$$
D\left(\widehat{H}_{0}\right)=C_{0}^{\infty}(\mathbb{R} \backslash\{0\}) .
$$


By the Krein's theory of s.a.e. for symmetric operators on Hilbert spaces one easily proves that there is a 4-parameter family of s.a.e. of (2.1). Besides, such a family can be translated into a 4-parameter family of boundary conditions at the point 0 . Finally, following [4] and [15] we know that any s.a.e. of $\widehat{H}_{0}$ can be described in one of the two following way:

- Given $\omega, a, b, c, d$ such that $|\omega|=1, a d-b c=1$, we define the s.a.e. $H_{U}$ as follows:

$$
\begin{aligned}
U & =\omega\left(\begin{array}{ll}
a & b \\
c & d
\end{array}\right), \\
D_{U}:=D\left(H_{U}\right) & =\left\{\psi \in H^{2}(\mathbb{R} \backslash\{0\}),\left(\begin{array}{c}
\psi(0+) \\
\psi^{\prime}(0+)
\end{array}\right)=U\left(\begin{array}{c}
\psi(0-) \\
\psi^{\prime}(0-)
\end{array}\right)\right\}, \\
\left(H_{U} \psi\right)(x) & =-\psi^{\prime \prime}(x), \quad x \neq 0, \quad \forall \psi \in D\left(H_{U}\right) .
\end{aligned}
$$

- Given $p, q \in \mathbb{R} \cup\{\infty\}$ we define the s.a.e $H_{p, q}$ as follows:

$$
\begin{aligned}
D_{p, q}:= & D\left(H_{p, q}\right)=\left\{\psi \in H^{2}(\mathbb{R} \backslash\{0\}), \psi(0+)=p \psi^{\prime}(0+), \quad \psi(0-)=q \psi^{\prime}(0-)\right\} \\
& \left(H_{p, q} \psi\right)(x)=-\psi^{\prime \prime}(x), \quad x \neq 0 \quad \forall \psi \in D\left(H_{p, q}\right)
\end{aligned}
$$

Remark 2.1. Using the description provided by the matrix $U$, and choosing $\omega=a=d=1$, $b=c=0$, one reconstructs the free-particle Hamiltonian $H_{0}$. Furthermore, the choice $\omega=a=d=1$, $b=0, c \neq 0$ corresponds to the well-known case of a pure Dirac's $\delta$ interaction of strength $c$. On the other hand, the case $\omega=a=d=1, c=0, b \in \mathbb{R}$ corresponds to the case of the so-called $\delta^{\prime}$ interaction of strength $b$, namely, to the boundary condition

$$
\begin{aligned}
\psi^{\prime}(0+) & =\psi^{\prime}(0-) \\
\psi(0+)-\psi(0-) & =b \psi^{\prime}(0-)
\end{aligned}
$$

Remark 2.2. The Neumann or Dirichlet boundary conditions in $0+$ are realized by choosing $p=\infty$ or $p=0$, respectively, and in $0-$ by choosing $q=\infty$ or $q=0$, respectively.

Preliminarily, we need to endow the space $D(H)$, domain of the Hamiltonian operator $H$, where $H$ is a general notation for $H_{U}$ and $H_{p, q}$, with the structure of a Hilbert space. To this aim, we first define the quantity

$$
m:=1-\inf \sigma(H) .
$$

From the general theory we know that $m$ is finite. Next, for any $\psi \in D(H)$ we introduce the norm

$$
\|\psi\|_{H}:=\|(H+m) \psi\|
$$

Notice that such a norm can be derived by a scalar product. Moreover, it is easily shown that it is equivalent to the standard graph norm.

In the following we investigate the problem of finding solutions to (1.1) in the form domain of the linear part of the generator. In order to do that we preliminarily describe such form domains. To this purpose we adapt the classification of form domains given in [22] to our context and our notation.

Proposition 2.3. The quadratic forms associated to the self-adjoint extensions of $\widehat{H}_{0}$ are defined as follows: 
1. For the Hamiltonian $H_{0,0}$ with Dirichlet boundary conditions, the energy space is

$$
Q_{0}:=\left\{\psi \in H^{1}(\mathbb{R}), \psi(0)=0\right\}
$$

and the form reads

$$
B_{0}(\psi)=\left\|\psi^{\prime}\right\|^{2} .
$$

2. For the Hamiltonian $H_{0, q}, q \neq 0$, with Dirichlet boundary conditions on $0+$ only, one has

$$
Q_{0+}:=\left\{\psi \in H^{1}\left(\mathbb{R}^{+}\right) \oplus H^{1}\left(\mathbb{R}^{-}\right), \psi(0+)=0\right\}
$$

and the form reads

$$
B_{0, q}(\psi)=\left\|\psi^{\prime}\right\|_{L^{2}\left(\mathbb{R}^{+}\right)}^{2}+\left\|\psi^{\prime}\right\|_{L^{2}\left(\mathbb{R}^{-}\right)}^{2}-|q|^{-1}|\psi(0-)|^{2} .
$$

Analogously,

$$
Q_{0-}:=\left\{\psi \in H^{1}\left(\mathbb{R}^{+}\right) \oplus H^{1}\left(\mathbb{R}^{-}\right), \psi(0-)=0\right\}
$$

and the form reads

$$
B_{p, 0}(\psi)=\left\|\psi^{\prime}\right\|_{L^{2}\left(\mathbb{R}^{+}\right)}^{2}+\left\|\psi^{\prime}\right\|_{L^{2}\left(\mathbb{R}^{-}\right)}^{2}+|p|^{-1}|\psi(0+)|^{2} .
$$

3. For the Hamiltonian $H_{U}$, defined in (2.3), with $b=0$ the energy space is

$$
Q_{\omega a}:=\left\{\psi \in H^{1}\left(\mathbb{R}^{+}\right) \oplus H^{1}\left(\mathbb{R}^{-}\right), \psi(0+)=\omega a \psi(0-)\right\}
$$

and the form reads

$$
B_{\omega a}(\psi)=\left\|\psi^{\prime}\right\|_{L^{2}\left(\mathbb{R}^{+}\right)}^{2}+\left\|\psi^{\prime}\right\|_{L^{2}\left(\mathbb{R}^{-}\right)}^{2}+a c|\psi(0-)|^{2}
$$

4. For any other s.a.e. of $\widehat{H}_{0}$ the energy space is given by

$$
Q:=H^{1}\left(\mathbb{R}^{+}\right) \oplus H^{1}\left(\mathbb{R}^{-}\right)
$$

To describe the action of the form we have to consider two cases:

(a) if the Hamiltonian is of the type $H_{U}$ described in (2.3), with $b \neq 0$, then

$$
B_{U}(\psi):=\left\|\psi^{\prime}\right\|_{L^{2}\left(\mathbb{R}^{+}\right)}^{2}+\left\|\psi^{\prime}\right\|_{L^{2}\left(\mathbb{R}^{-}\right)}^{2}+b^{-1}\left[d|\psi(0+)|^{2}+a|\psi(0-)|^{2}-2 \operatorname{Re}(\omega \overline{\psi(0+)} \psi(0-))\right]
$$

(b) if the Hamiltonian is of the type $H_{p, q}$ described in (2.4), with $p, q$ both different from zero, then

$$
B_{p, q}(\psi):=\left\|\psi^{\prime}\right\|_{L^{2}\left(\mathbb{R}^{+}\right)}^{2}+\left\|\psi^{\prime}\right\|_{L^{2}\left(\mathbb{R}^{-}\right)}^{2}+p^{-1}|\psi(0+)|^{2}-q^{-1}|\psi(0-)|^{2}
$$

Remark 2.4. For a pure Dirac's $\delta$ interaction formula (2.15) applies. The form domain coincides with $H^{1}(\mathbb{R})$ and the action of the form reads

$$
B_{1}(\psi)=\left\|\psi^{\prime}\right\|^{2}+c|\psi(0)|^{2} .
$$


Remark 2.5. In the case 4 . the form domain can be represented by the sum of a regular part and a jump:

$$
Q:=\left\{\psi \in H^{1}(\mathbb{R} \backslash\{0\})\right\}=\left\{\psi \in H^{1}(\mathbb{R} \backslash\{0\}), \psi=\phi+q \frac{\epsilon}{2}, \phi^{\prime} \in L^{2}(\mathbb{R}), q \in \mathbb{C}\right\}
$$

where $\epsilon$ denotes the sign function. Notice that

$$
q=\psi(0+)-\psi(0-)
$$

For a pure $\delta^{\prime}$ interaction one has

$$
B(\psi):=\left\|\phi^{\prime}\right\|^{2}+b^{-1}|q|^{2}
$$

Besides, if $\psi$ belongs to the operator domain of a $\delta^{\prime}$-interaction then

$$
B(\psi):=\left\|\phi^{\prime}\right\|^{2}+b\left|\psi^{\prime}(0)\right|^{2}
$$

where, with a slight abuse of notation, we denoted by $\psi^{\prime}(0)$ the quantity $\psi^{\prime}(0+)=\psi^{\prime}(0-)$.

Remark 2.6. In the following we will frequently use the decomposition of the elements of the form domain introduced in (2.19).

Obviously, such a decomposition holds for all other energy spaces too. Notice that for the elements of $Q_{0}$ and $Q_{1}\left(=H^{1}(\mathbb{R})\right)$ one has $q=0$.

We will usually refer to $\phi$ as to the regular part, to $\frac{q \epsilon}{2}$ as to the singular part, to $q$ as to the jump of $\psi$. Notice that if $q \neq 0$ then the function $\phi$ is not square integrable, even though it is locally $H^{1}$ : indeed, at least one of the two quantities $\lim _{x \rightarrow \pm \infty} \phi(x)$ is different from zero.

All energy spaces can be endowed with the structure of Hilbert space by introducing the norm

$$
\|\psi\|_{X}^{2}=\|\psi\|^{2}+\lim _{\varepsilon \rightarrow 0+} \int_{\varepsilon}^{+\infty}\left|\psi^{\prime}(x)\right|^{2} d x+\lim _{\varepsilon \rightarrow 0+} \int_{-\infty}^{-\varepsilon}\left|\psi^{\prime}(x)\right|^{2} d x
$$

where $X$ denotes any form domain introduced in proposition 2.3. It is immediately seen that such a norm is induced by the scalar product

$$
(\psi, \phi)_{X}=(\psi, \phi)+\lim _{\varepsilon \rightarrow 0+} \int_{\varepsilon}^{+\infty} \overline{\psi(x)} \phi(x) d x+\lim _{\varepsilon \rightarrow 0+} \int_{-\infty}^{-\varepsilon} \overline{\psi(x)} \phi(x) d x .
$$

In proposition 5.2 we prove that the norm defined in (2.23) is equivalent to the following energy norm

$$
\|\psi\|_{B}^{2}:=B(\psi)+m\|\psi\|^{2}
$$

where $B$ is any form introduced in proposition 2.3 and the quantity $m$ was defined in (2.6).

\subsection{Dual of energy spaces}

In the proof of the well-posedness for problem (1.2) in the energy domain we need to know the duals of the energy spaces (at least in the case 3. of proposition 2.3). By definitions (2.8), (2.16), (2.14) one has

$$
Q_{0} \subset Q_{\omega a} \subset Q, \quad \omega \in \mathbb{C},|\omega|=1, a \in \mathbb{R} .
$$


Proposition 2.7. The following orthogonal decompositions hold

$$
\begin{aligned}
Q_{\omega a} & =Q_{0} \oplus_{\perp} \operatorname{Span}\left(\varphi_{\omega a}\right) \\
Q & =Q_{\omega a} \oplus_{\perp} \operatorname{Span}\left(\varphi_{-\omega a^{-1}}\right)=Q_{0} \oplus_{\perp} \operatorname{Span}\left(\varphi_{+}, \varphi_{-}\right)
\end{aligned}
$$

where the notation $\varphi_{0}, \varphi_{ \pm}$where introduced in section 1.1 .

Proof. One can decompose any $\psi \in Q_{\omega a}$ as follows:

$$
\psi=\xi+\psi(0+) \varphi_{+}+\psi(0-) \varphi_{-}=\xi+\psi(0-) \varphi_{\omega a} .
$$

Obviously $\xi$ belongs to $Q_{0}$. One can directly verify orthogonality by performing the scalar products, as defined in (2.24).

Now the duals of form domains in proposition 2.3 can be easily computed.

Proposition 2.8. The following relationships hold between the spaces $Q_{0}^{\star}, Q_{\omega a}^{\star}$, and $Q^{\star}$ :

$$
\begin{aligned}
Q_{\omega a}^{\star} & =Q^{\star} / Q_{\omega a}^{\perp} \\
Q_{0}^{\star} & =Q_{\omega a}^{\star} / Q_{0}^{\perp}=Q^{\star} / Q_{0}^{\perp}
\end{aligned}
$$

where, given $Y \subset X$, the space $Y^{\perp} \subset X^{\star}$ is defined as follows:

$$
Y^{\perp}:=\left\{f \in X^{\star}, \text { s.t. }\langle f, y\rangle_{X^{\star}, X}=0, \quad \forall y \in Y\right\}
$$

Proof. The proposition immediately follows from a classical result in functional analysis (see e.g. [26], sec. 4.5).

Proposition 2.9. The spaces $Q_{0}^{\star}, Q_{\omega a}^{\star}$, and $Q^{\star}$ can be represented as follows:

$$
\begin{aligned}
Q_{0}^{\star} & =\left\{f \in H^{-1}(\mathbb{R}), \text { s.t. } \int_{\mathbb{R}} \frac{\widehat{f}(k)}{k^{2}+1} d k=0\right\} \\
Q_{\omega a}^{\star} & =Q_{0}^{\star} \oplus \operatorname{Span}\left(\delta^{\omega a}(0+)\right) \\
Q^{\star} & =Q_{0}^{\star} \oplus \operatorname{Span}(\delta(0+), \delta(0-))
\end{aligned}
$$

We used the notation $\delta(0 \pm)$ to denote the functionals acting as follows:

$$
\langle\delta(0 \pm), \psi\rangle_{X^{\star}, X}=\lim _{x \rightarrow 0 \pm} \psi(x)
$$

with the quotient notation for duals introduced in (2.30).

Besides, we denoted $\delta^{\omega a}(0+)$ the functional that vanishes on $Q_{0} \oplus \operatorname{Span}\left(\varphi_{-\omega a^{-1}}\right)$ and gives $\omega a$ when acting on $\varphi_{\omega a}$.

Proof. From the first decomposition in proposition 2.7, choosing $\omega=1, a=1$, we obtain

$$
H^{1}(\mathbb{R})=Q_{0} \oplus_{\perp} \operatorname{Span}\left(\varphi_{1}\right) .
$$

Then, the second formula in proposition 2.8 provides

$$
Q_{0}^{\star}=H^{-1}(\mathbb{R}) / Q_{0}^{\perp} .
$$


From elementary linear algebra we know that the dimension of $Q_{0}^{\perp}$ as a subspace of $H^{-1}(\mathbb{R})$ is given by

$$
\operatorname{dim}\left[\left(Q_{0}^{\perp}\right)_{H^{-1}(\mathbb{R})}\right]=\operatorname{dim}\left(H^{1}(\mathbb{R}) / Q_{0}\right)=1 .
$$

Since $\delta(0)$ belongs to the one-dimensional space $\left(Q_{0}^{\perp}\right)_{H^{-1}(\mathbb{R})}$, it spans the whole space. So formula (2.31) is proven.

Imposing the constraint that any element $f$ of $Q_{0}^{\star}$ must be orthogonal to the function $e^{-|\cdot|}$ one obtains the representation in the Fourier space (2.31).

Now we prove formula (2.32). First, reasoning as in the previous case it is immediately seen that

$$
\operatorname{dim}\left[\left(Q_{0}^{\perp}\right)_{Q_{\omega a}^{\star}}\right]=\operatorname{dim}\left(Q_{\omega a} / Q_{0}\right)=1 .
$$

Since $\delta^{\omega a}(0+)$ belongs to the one-dimensional space $\left(Q_{0}^{\perp}\right)_{Q_{\omega a}^{\star}}$, it spans the whole space. So formula (2.32) is proven.

The proof of (2.33) is analogous. So we proved proposition 2.9.

Corollary 2.10 (Decomposition of dual spaces). For any $f \in Q^{\star}$ there exist $f_{0} \in Q_{0}^{\star}, f_{\omega a} \in Q_{\omega a}^{\star}$, $\alpha, \beta \in \mathbb{C}$, such that:

$$
\begin{aligned}
& f=f_{0}+\alpha \delta(0+)+\beta \delta(0-) \\
& f=f_{\omega a}+\frac{\beta-\omega a^{-1} \alpha}{1+a^{2}}\left[a^{2} \delta(0-)-\bar{\omega} a \delta(0+)\right]
\end{aligned}
$$

Proof. Let $f$ be an element of $Q^{\star}$. For any $\psi \in Q$ define

$$
f_{0}(\psi):=f\left(\psi_{0}\right)
$$

where $\psi_{0}$ is the $H_{0}^{1}$-component of $\psi$ according to decomposition (2.27). Moreover, define

$$
\alpha:=f\left(\varphi_{+}\right), \quad \beta:=f\left(\varphi_{-}\right)
$$

and decomposition (2.34) is proven. Furthermore, we define the functional $f_{\omega a}$ as follows:

$$
f_{\omega a}:=f_{0}(\psi)+\frac{\omega a \alpha+\beta}{1+a^{2}}[\bar{\omega} a \delta(0+)+\delta(0-)]
$$

To see that $f_{\omega a}$ is indeed in $Q_{\omega a}^{\star}$ it is sufficient to show that it is orthogonal to $Q_{-\omega a^{-1}}$, where orthogonality is to be understood in the sense of (2.30). But this is straightforward: since such orthogonal space must be one-dimensional (see (2.27)), so it must be spanned by the function $\varphi_{-\omega a^{-1}}$, which is manifestly orthogonal to $f_{w a}$. So $(2.35)$ is proven.

Corollary 2.11. The functional $f_{1}$ reads as follows:

$$
f_{1}(\psi):=f_{0}(\psi)+\frac{1}{2}(\alpha+\beta)(\psi(0+)+\psi(0-))
$$

and the related decomposition gives

$$
f=f_{1}+\frac{\beta-\alpha}{2}[\delta(0-)-\delta(0+)] .
$$


Remark 2.12. Sometimes the correspondence between the functional $f$ and its $Q_{0}^{\star}$ or $H^{-1}$ component may be not so evident. For instance, consider the functional $f^{\eta} \in Q^{\star}$, defined by

$$
\left\langle f^{\eta}, \psi\right\rangle_{Q^{\star}, Q}:=(\eta, \psi),
$$

where $\eta \in C_{0}^{\infty} \backslash\{0\}$. Even though represented by a smooth function in $Q_{0}$, it has non-trivial $\delta(0+)$ and $\delta(0-)$ components. Indeed, applying $f^{\eta}$ to any function $\psi \in Q$ one gets

$$
\left\langle f^{\eta}, \psi\right\rangle_{Q^{\star}, Q}:=\left(\eta, \psi_{0}\right)+\psi(0+)\left(\eta, \varphi_{+}\right)+\psi(0-)\left(\eta, \varphi_{-}\right)
$$

and therefore the functional $f^{\eta}$ decomposes as follows:

$$
f^{\eta}=f_{0}^{\eta}+\left(\eta, \varphi_{+}\right) \delta(0+)+\left(\eta, \varphi_{-}\right) \delta(0-)
$$

where $f^{\eta}$ acts on $Q_{0}$ as the scalar product in $L^{2}$ with the function $\eta$, and vanishes on $Q_{0}^{\perp}$.

Definition 2.13. For any energy domain $X$ we define the norm in $X^{\star}$ by

$$
\|f\|_{X^{\star}}:=\sup _{\psi \in X \backslash\{0\}} \frac{\left|\langle f, \psi\rangle_{X^{\star}, X}\right|}{\|\psi\|_{X}} .
$$

Remark 2.14. It appears that, for any $f \in X^{\star}, \omega \in \mathbb{C}$ with $|\omega|=1$, and $a \in \mathbb{R}$,

$$
\left\|f_{0}\right\|_{Q_{0}^{\star}} \leq\left\|f_{\omega a}\right\|_{Q_{\omega a}^{\star}} \leq\|f\|_{Q^{\star}}
$$

Vice versa, given $f \in Q_{0}^{\star}$, it is possible to define its trivial extension $\tilde{f}$ to $Q_{\omega a}$ as the functional acting like $f$ on $Q_{0}$ and vanishing on $\varphi_{\omega a}$. Obviously, it can be further extended to $\widetilde{f}$, that vanishes on $\varphi_{-\omega a^{-1}}$. One has

$$
\|f\|_{Q_{0}^{\star}}=\|\tilde{f}\|_{Q_{\omega a}^{\star}}=\|\tilde{f}\|_{Q^{\star}} .
$$

\section{Estimates}

In this section we prove estimates for functions in the energy spaces. We give results for functions in $Q$, but it is obvious that they hold for the other form domains introduced in proposition 2.3 too.

First of all, we introduce a decomposition for the elements of $Q$, that in the sequel will prove useful.

Lemma 3.1. For any function $\psi \in Q$, there exists a unique couple of even functions $\psi_{+}, \psi_{-} \in H^{1}(\mathbb{R})$ such that

$$
\psi(x)=\chi_{+}(x) \psi_{+}(x)+\chi_{-}(x) \psi_{-}(x),
$$

Proof. Given $\psi \in Q$ we define the functions $\psi_{+}, \psi_{-}$as follows:

$$
\psi_{ \pm}(x):=\left\{\begin{array}{cc}
\psi( \pm x), & x \in(0,+\infty) \\
\psi(0 \pm), & x=0 \\
\psi(\mp x), & x \in(-\infty, 0)
\end{array}\right.
$$

It is immediately seen that both functions $\psi_{ \pm}$belong to $H^{1}(\mathbb{R})$ and satisfy (3.1). Any other decomposition in even functions lying in $H^{1}$ must fulfil (3.2), so uniqueness is proven. 
Remark 3.2. The following formulae link decomposition (3.1) to the one introduced in (2.19):

$$
\begin{aligned}
\phi(x) & =\chi_{+}(x)\left(\psi_{+}(x)-\frac{q}{2}\right)+\chi_{-}(x)\left(\psi_{-}(x)+\frac{q}{2}\right) \\
\phi^{\prime}(x) & =\chi_{+}(x) \psi_{+}^{\prime}(x)+\chi_{-}(x) \psi_{-}^{\prime}(x) \\
q & =\psi_{+}(0)-\psi_{-}(0)
\end{aligned}
$$

Remark 3.3. As a consequence, the following estimates hold:

$$
\begin{aligned}
\|\psi\|^{2} & =\frac{1}{2}\left\|\psi_{+}\right\|^{2}+\frac{1}{2}\left\|\psi_{-}\right\|^{2} \\
\left\|\phi^{\prime}\right\|^{2} & =\frac{1}{2}\left\|\psi_{+}^{\prime}\right\|^{2}+\frac{1}{2}\left\|\psi_{-}^{\prime}\right\|^{2} \\
\left\|\psi_{ \pm}^{\prime}\right\| & \leq \sqrt{2}\left\|\phi^{\prime}\right\| \\
|q| & \leq \frac{1}{\sqrt{2}}\left\|\psi_{+}-\psi_{-}\right\|_{H^{1}} \leq \frac{1}{\sqrt{2}}\left(\left\|\psi_{+}\right\|_{H^{1}}+\left\|\psi_{-}\right\|_{H^{1}}\right)
\end{aligned}
$$

Remark 3.4. The norm introduced in (2.23) for elements of the energy space can be expressed as follows:

$$
\|\psi\|_{Q}^{2}:=\frac{1}{2}\left\|\psi_{+}\right\|_{H^{1}}^{2}+\frac{1}{2}\left\|\psi_{-}\right\|_{H^{1}}^{2}=\|\psi\|^{2}+\left\|\phi^{\prime}\right\|^{2}
$$

Remark 3.5. From the previous remarks we know that

$$
\begin{aligned}
\|\psi\|_{\infty} & \leq C\|\psi\|_{Q} \\
\left\|\phi^{\prime}\right\| & \leq C\|\psi\|_{Q} \\
|q| & \leq C\|\psi\|_{Q} .
\end{aligned}
$$

Therefore, using (3.3),

$$
\|\phi\|_{\infty} \leq C\|\psi\|_{Q}
$$

Notice that, since the $H$-norm is stronger than the $Q$-norm, all these estimates hold in $D(H)$ too. $Q$.

We prove a lemma that extends the one-dimensional Gagliardo-Nirenberg's estimate to the space

Lemma 3.6. For any $p \in(2,+\infty]$ there exists $C_{p}>0$ such that, for any $\psi \in Q, \psi=\phi+q \frac{\epsilon}{2}$,

$$
\|\psi\|_{p} \leq C_{p}\left\|\phi^{\prime}\right\|^{\frac{1}{2}-\frac{1}{p}}\|\psi\|^{\frac{1}{2}+\frac{1}{p}}
$$

Proof. Let $\psi$ be an element of $Q$. Using lemma 3.1, we can write $\psi$ as

$$
\psi=\chi_{+} \psi_{+}+\chi_{-} \psi_{-}
$$

Then,

$$
\|\psi\|_{p} \leq C_{p}\left(\left\|\psi_{+}\right\|_{p}+\left\|\psi_{-}\right\|_{p}\right)
$$

Applying standard Gagliardo-Nirenberg's inequality and recalling (3.8)

$$
\begin{aligned}
\|\psi\|_{p} & \leq C_{p}\left(\left\|\psi_{+}^{\prime}\right\|^{\frac{1}{2}-\frac{1}{p}}\left\|\psi_{+}\right\|^{\frac{1}{2}+\frac{1}{p}}+\left\|\psi_{-}^{\prime}\right\|^{\frac{1}{2}-\frac{1}{p}}\left\|\psi_{-}\right\|^{\frac{1}{2}+\frac{1}{p}}\right) \\
& \leq C_{p}\left\|\phi^{\prime}\right\|^{\frac{1}{2}-\frac{1}{p}}\left(\left\|\psi_{+}\right\|^{\frac{1}{2}+\frac{1}{p}}+\left\|\psi_{-}\right\|^{\frac{1}{2}+\frac{1}{p}}\right) \\
& \leq C_{p}\left\|\phi^{\prime}\right\|^{\frac{1}{2}-\frac{1}{p}}\|\psi\|^{\frac{1}{2}+\frac{1}{p}}
\end{aligned}
$$

and the lemma is proven. 
Remark 3.7. In particular, for $p=\infty$ we obtain the generalization to $Q$ of the embedding of $H^{1}$ in $L^{\infty}$ :

Furthermore, by (3.13),

$$
\|\psi\|_{\infty} \leq C\left\|\phi^{\prime}\right\|^{\frac{1}{2}}\|\psi\|^{\frac{1}{2}}
$$

$$
|q| \leq C\left\|\phi^{\prime}\right\|^{\frac{1}{2}}\|\psi\|^{\frac{1}{2}}
$$

\section{Global well-posedness in $\mathrm{D}(\mathrm{H})$}

In this section we prove existence and uniqueness for the problem (1.2) in the operator domain of $H$. Furthermore, we show that $L^{2}$-norm and energy are conserved quantities and use this result to prove that all maximal solutions are global in time (i.e. there is no blow-up phenomenon).

Theorem 4.1 (Existence and uniqueness for solutions in $D(H)$ ). Let $H$ be any self-adjoint extension of the operator $\widehat{H}_{0}$ defined in $(2.1),(2.2)$. Let its domain be denoted by $D(H)$.

For any $\psi_{0} \in D(H)$ there exists $T \in(0,+\infty)$ such that the equation (1.2) has a unique solution $\psi \in C([0, T), D(H)) \cap C^{1}\left([0, T), L^{2}(\mathbb{R})\right)$.

Moreover, equation (1.2) has a maximal solution $\psi(t)$ defined on the interval $\left[0, T^{\star}\right)$. For such a solution, the following alternative holds: either $T^{\star}=+\infty$ or

$$
\lim _{t \rightarrow T^{\star}}\|\psi(t)\|_{H}=+\infty \text {. }
$$

Proof. Let us use the notation

$$
\mathcal{X}=C^{0}([0, T], D(H)) \cap C^{1}\left([0, T], L^{2}\left(\mathbb{R}^{3}\right)\right)
$$

and provide the space $\mathcal{X}$ with the norm

$$
\|\psi\|_{\mathcal{X}}:=\max _{t \in[0, T]}\|\psi(t)\|_{H}+\max _{t \in[0, T]}\left\|\partial_{t} \psi(t)\right\|
$$

where the norm $\|\cdot\|_{H}$ has been defined in (2.7).

Given $\psi_{0} \in D(H)$, we define the function $G: \mathcal{X} \rightarrow \mathcal{X}$, as

$$
G \psi:=e^{-i H \cdot} \psi_{0}-i \lambda \int_{0} d s e^{-i(\cdot-s) H}|\psi(s)|^{2} \psi(s)
$$

First, it is immediately seen that

$$
\left\|e^{-i H t} \psi_{0}\right\|_{H}=\left\|\psi_{0}\right\|_{H}, \quad\left\|\partial_{t} e^{-i H t} \psi_{0}\right\|=\left\|H \psi_{0}\right\|
$$

Therefore,

$$
\left\|e^{-i H t} \psi_{0}\right\|_{\mathcal{X}}=\left\|\psi_{0}\right\|_{H}+\left\|H \psi_{0}\right\| \leq 2\left\|\psi_{0}\right\|_{H} .
$$

Next, integrating by parts we obtain the identity

$$
\begin{aligned}
& \int_{0}^{t} d s e^{-i H(t-s)}|\psi(s)|^{2} \psi(s) \\
= & -i(H+m)^{-1}|\psi(t)|^{2} \psi(t)+i e^{-i H t}(H+m)^{-1}\left|\psi_{0}\right|^{2} \psi_{0}+m(H+m)^{-1} \int_{0}^{t} d s e^{-i H(t-s)}|\psi(s)|^{2} \psi(s) \\
& +2 i(H+m)^{-1} \int_{0}^{t} d s e^{-i H(t-s)}|\psi(s)|^{2} \partial_{s} \psi(s)+i(H+m)^{-1} \int_{0}^{t} d s e^{-i H(t-s)} \psi^{2}(s) \partial_{s} \bar{\psi}(s) \\
& =(I)_{t}+(I I)_{t}+(I I I)_{t}+(I V)_{t}+(V)_{t}
\end{aligned}
$$


Furthermore,

$$
\begin{aligned}
\left\|(I)_{t}\right\|_{H} & \leq\|\psi(t)\|_{\infty}^{2}\|\psi(t)\| \leq C\|\psi(t)\|_{H}^{3} \\
\left\|(I I)_{t}\right\|_{H} & \leq C\left\|\psi_{0}\right\|_{H}^{3} \\
\left\|(I I I)_{t}\right\|_{H} & =|m|\left\|\int_{0}^{t} d s e^{-i H(t-s)}|\psi(s)|^{2} \psi(s)\right\| \leq C T \max _{t \in[0, T]}\|\psi(t)\|_{H}^{3} \\
\left\|(I V)_{t}\right\|_{H} & =2\left\|\int_{0}^{t} d s e^{-i H(t-s)}|\psi(s)|^{2} \partial_{s} \psi(s)\right\| \leq C T \max _{t \in[0, T]}\left(\|\psi(t)\|_{H}^{2}\left\|\partial_{t} \psi(t)\right\|\right) \\
\left\|(V)_{t}\right\|_{H} & \leq C T \max _{t \in[0, T]}\left(\|\psi(t)\|_{H}^{2}\left\|\partial_{t} \psi(t)\right\|\right)
\end{aligned}
$$

Then,

$$
\left\|\int_{0}^{t} d s e^{-i H(t-s)}|\psi(s)|^{2} \psi(s)\right\|_{H} \leq C T\|\psi\|_{\mathcal{X}}^{3}
$$

Now we estimate the $L^{2}$-norm of the time derivative of terms $(I)_{t},(I I)_{t},(I I I)_{t},(I V)_{t},(V)_{t}$.

$$
\begin{aligned}
\left\|\partial_{t}(I)_{t}\right\| & =\left\|(H+m)^{-1}\left(2|\psi(t)|^{2} \partial_{t} \psi(t)+\psi^{2}(t) \partial_{t} \bar{\psi}(t)\right)\right\| \leq C\|\psi(t)\|_{H}^{2}\left\|\partial_{t} \psi(t)\right\| \\
\left\|\partial_{t}(I I)_{t}\right\| & \leq\left\|\psi_{0}\right\|_{H}^{3} \\
\left\|\partial_{t}(I I I)_{t}\right\| & =\left\|m(H+m)^{-1}|\psi(t)|^{2} \psi(t)-i m \int_{0}^{t} d s e^{-i H(t-s)} H(H+m)^{-1}|\psi(s)|^{2} \psi(s)\right\| \\
& \leq C\|\psi(t)\|_{H}^{2}\|\psi(t)\|+C T \max _{t \in[0, T]}\|\psi(t)\|_{H}^{2}\|\psi(t)\| \\
\left\|\partial_{t}(I V)_{t}\right\| & \leq C\left\|(H+m)^{-1}|\psi(t)|^{2} \partial_{t} \psi(t)\right\|+2 \int_{0}^{t} d s\left\|e^{-i H(t-s)} H(H+m)^{-1}|\psi(s)|^{2} \partial_{s} \psi(s)\right\| \\
& \leq C\|\psi(t)\|_{H}^{2}\left\|\partial_{t} \psi(t)\right\|+C T \max _{t \in[0, T]}\left(\|\psi(t)\|_{H}^{2}\left\|\partial_{t} \psi(t)\right\|\right) \\
\left\|\partial_{t}(V)_{t}\right\| & \leq C\|\psi(t)\|_{H}^{2}\left\|\partial_{t} \psi(t)\right\|+C T \max _{t \in[0, T]}\left(\|\psi(t)\|_{H}^{2}\left\|\partial_{t} \psi(t)\right\|\right)
\end{aligned}
$$

Therefore

$$
\left\|\partial_{t}\left[\int_{0}^{t} d s e^{-i H(t-s)}|\psi(s)|^{2} \psi(s)\right]\right\| \leq C T\|\psi\|_{\mathcal{X}}^{3}
$$

and from (4.8), (4.10) and (4.5) one finally finds

$$
\|G \psi\|_{\mathcal{X}} \leq 2\left\|\psi_{0}\right\|_{H}+C T\|\psi\|_{\mathcal{X}}^{3}
$$

Now let us consider two elements of $\mathcal{X}$, say $\psi$ and $\xi$. As before, integrating by parts

$$
\begin{aligned}
& \int_{0}^{t} d s e^{-i H(t-s)}\left(|\psi(s)|^{2} \psi(s)-|\xi(s)|^{2} \xi(s)\right) \\
= & -i(H+m)^{-1}\left(|\psi(t)|^{2} \psi(t)-|\xi(t)|^{2} \xi(t)\right)+i e^{-i H t}(H+m)^{-1}\left(\left|\psi_{0}\right|^{2} \psi_{0}-\left|\xi_{0}\right|^{2} \xi_{0}\right) \\
& +m(H+m)^{-1} \int_{0}^{t} d s e^{-i H(t-s)}\left(|\psi(s)|^{2} \psi(s)-|\xi(s)|^{2} \xi(s)\right) \\
& +2 i(H+m)^{-1} \int_{0}^{t} d s e^{-i H(t-s)}\left(|\psi(s)|^{2} \partial_{s} \psi(s)-|\xi(s)|^{2} \partial_{s} \xi(s)\right) \\
& +i(H+m)^{-1} \int_{0}^{t} d s e^{-i H(t-s)}\left(\psi^{2}(s) \partial_{s} \bar{\psi}(s)-\xi^{2}(s) \partial_{s} \bar{\xi}(s)\right) \\
& =(V I)_{t}+(V I I)_{t}+(V I I I)_{t}+(I X)_{t}+(X)_{t}
\end{aligned}
$$


Now,

$$
\begin{aligned}
\left\|(V I)_{t}\right\|_{H} & =\left\||\psi(t)|^{2} \psi(t)-|\xi(t)|^{2} \xi(t)\right\| \leq C\left(\|\psi(t)\|_{H}^{2}+\|\xi(t)\|_{H}^{2}\right)\|\psi(t)-\xi(t)\|_{H} \\
& \leq C\left(\|\psi\|_{\mathcal{X}}^{2}+\|\xi\|_{\mathcal{X}}^{2}\right)\|\psi-\xi\|_{\mathcal{X}} \\
\left\|(V I I)_{t}\right\|_{H} & \leq C\left(\|\psi\|_{\mathcal{X}}^{2}+\|\xi\|_{\mathcal{X}}^{2}\right)\|\psi-\xi\|_{\mathcal{X}} \\
\left\|(V I I I)_{t}\right\|_{H} & \leq m\left\|\int_{0}^{t} d s e^{-i H(t-s)}\left(\mid \psi^{2}(s) \bar{\psi}(s)-\xi^{2}(s) \bar{\xi}(s)\right)\right\| \leq C T\left(\|\psi\|_{\mathcal{X}}^{2}+\|\xi\|_{\mathcal{X}}^{2}\right)\|\psi-\xi\|_{\mathcal{X}} \\
\left\|(I X)_{t}\right\|_{H} & \leq 2\left\|\int_{0}^{t} d s e^{-i H(t-s)}\left(\mid \psi^{2}(s) \partial_{s} \bar{\psi}(s)-\xi^{2}(s) \partial_{s} \bar{\xi}(s)\right)\right\| \leq C T\left(\|\psi\|_{\mathcal{X}}^{2}+\|\xi\|_{\mathcal{X}}^{2}\right)\|\psi-\xi\|_{\mathcal{X}} \\
\left\|(X)_{t}\right\|_{H} & \leq C T\left(\|\psi\|_{\mathcal{X}}^{2}+\|\xi\|_{\mathcal{X}}^{2}\right)\|\psi-\xi\|_{\mathcal{X}}
\end{aligned}
$$

Then,

$$
\left\|\int_{0}^{t} d s e^{-i H(t-s)}\left(|\psi(s)|^{2} \psi(s)-|\xi(s)|^{2} \xi(s)\right)\right\|_{H} \leq C T\left(\|\psi\|_{\mathcal{X}}^{2}+\|\xi\|_{\mathcal{X}}^{2}\right)\|\psi-\xi\|_{\mathcal{X}}
$$

Moreover,

$$
\begin{aligned}
\left\|\partial_{t}(V I)_{t}\right\|_{H} & \leq C\left\||\psi(t)|^{2} \partial_{t} \psi(t)-|\xi(t)|^{2} \partial_{t} \xi(t)\right\|+\left\|\psi^{2}(t) \partial_{t} \bar{\psi}(t)-\xi^{2}(t) \partial_{t} \bar{\xi}(t)\right\| \\
& \leq C\left(\|\psi\|_{\mathcal{X}}^{2}+\|\xi\|_{\mathcal{X}}^{2}\right)\|\psi-\xi\|_{\mathcal{X}} \\
\left\|\partial_{t}(V I I)_{t}\right\| & \leq C\left(\|\psi\|_{\mathcal{X}}^{2}+\|\xi\|_{\mathcal{X}}^{2}\right)\|\psi-\xi\|_{\mathcal{X}} \\
\left\|\partial_{t}(V I I I)_{t}\right\| & \leq C\left(\|\psi\|_{\mathcal{X}}^{2}+\|\xi\|_{\mathcal{X}}^{2}\right)\|\psi-\xi\|_{\mathcal{X}}+C T\left(\|\psi\|_{\mathcal{X}}^{2}+\|\xi\|_{\mathcal{X}}^{2}\right)\|\psi-\xi\|_{\mathcal{X}} \\
\left\|\partial_{t}(I X)_{t}\right\| & \leq C\left\||\psi(t)|^{2} \partial_{t} \psi(t)-|\xi(t)|^{2} \partial_{t} \xi(t)\right\|+C \int_{0}^{t} d s\left\|\left.\psi(s)\right|^{2} \partial_{s} \psi(s)-|\xi(s)|^{2} \partial_{t} \xi(s)\right\| \\
& \leq C T\left(\|\psi\|_{\mathcal{X}}^{2}+\|\xi\|_{\mathcal{X}}^{2}\right)\|\psi-\xi\|_{\mathcal{X}} \\
\left\|\partial_{t}(X)_{t}\right\| & \leq C T\left(\|\psi\|_{\mathcal{X}}^{2}+\|\xi\|_{\mathcal{X}}^{2}\right)\|\psi-\xi\|_{\mathcal{X}}
\end{aligned}
$$

Then,

$$
\left\|\partial_{t} \int_{0}^{t} d s e^{-i H(t-s)}\left(|\psi(s)|^{2} \psi(s)-|\xi(s)|^{2} \xi(s)\right)\right\| \leq C T\left(\|\psi\|_{\mathcal{X}}^{2}+\|\xi\|_{\mathcal{X}}^{2}\right)\|\psi-\xi\|_{\mathcal{X}}
$$

From (4.14) and (4.16)

$$
\|G \psi-G \xi\|_{\mathcal{X}} \leq C T\left(\|\psi\|_{\mathcal{X}}^{2}+\|\xi\|_{\mathcal{X}}^{2}\right)\|\psi-\xi\|_{\mathcal{X}}
$$

Let us fix $M:=4\left\|\psi_{0}\right\|_{H}$ and consider the ball of radius $M$ in the space $\mathcal{X}$, namely

$$
\mathcal{Y}:=\left\{\psi \in \mathcal{X},\|\psi\|_{\mathcal{X}} \leq M\right\}
$$

Obviously, $\mathcal{Y}$ is a complete metric space with the norm induced by $\mathcal{X}$. From (4.11) and (4.17) one has that

$$
\begin{aligned}
\|G \psi\|_{\mathcal{X}} & \leq \frac{M}{2}+C T M^{3} \\
\|G \psi-G \xi\|_{\mathcal{X}} & \leq C M^{2} T\|\psi-\xi\|_{\mathcal{X}}
\end{aligned}
$$


If one chooses $T=\left(2 C M^{2}\right)^{-1}$, then $G$ is a contraction in $\mathcal{Y}$. By Banach-Caccioppoli's contraction lemma we immediately obtain the well-posedness of the problem (1.2) in $D(H)$. The blow-up alternative follows from the fact that the size of the interval of existence of the solution is a function of the $H$-norm of the solution.

The theorem is proven.

Remark 4.2. Actually, existence and uniqueness hold in the space $C([0, T), D(H))$, as appears from the fact that the time derivative of any solution in such space must belong to $C\left([0, T), L^{2}(\mathbb{R})\right)$.

Corollary 4.3 (Local existence and uniqueness for strong solutions). Let $H$ be any s.a.e. for the operator $\widehat{H}_{0}$ defined in (2.1), (2.2). Then, for any $\psi_{0} \in D(H)$ there exists $T \in(0,+\infty)$ s.t. the initial value problem

$$
\left\{\begin{array}{ccc}
i \partial_{t} \psi(t) & = & H \psi(t)+\lambda|\psi(t)|^{2} \psi(t) \\
\psi(0) & = & \psi_{0}
\end{array}\right.
$$

has a unique solution $\psi \in C([0, T), D(H)) \cap C^{1}\left([0, T), L^{2}(\mathbb{R})\right)$.

Proof. The solution of the integral equation (1.2), whose existence has been shown in theorem 4.1, solves (4.20) too.

Proposition 4.4 (Conservation laws for strong solutions). For any solution $\psi \in C([0, T), D(H))$ to the problem (1.2) the following conservation laws hold at any time $t$ in the interval $[0, T)$ :

$$
\begin{aligned}
\|\psi(t)\| & =\left\|\psi_{0}\right\| \\
\mathcal{E}[\psi(t)] & =\mathcal{E}\left[\psi_{0}\right]
\end{aligned}
$$

where the energy functional is defined as

$$
\mathcal{E}[\psi]=B(\psi)+\frac{\lambda}{2}\|\psi\|_{4}^{4}
$$

and $B$ is the quadratic form associated to the operator $H$.

Proof. Let $\psi_{0}$ be an element of $D(H)$, and $\psi(t)$ the solution to (4.20) at time $t$.

The conservation law for the $L^{2}$-norm can be immediately obtained recalling that

$$
\partial_{t}\|\psi(t)\|^{2}=2 \operatorname{Re}\left(\psi(t), \partial_{t} \psi(t)\right)
$$

and applying (4.20).

For the conservation of energy, first we prove that for any $t \in(0, T)$ the quantity $(\psi(t), H \psi(t))$ is differentiable and

$$
\partial_{t}(\psi(t), H \psi(t))=2 \operatorname{Re}\left(\partial_{t} \psi(t), H \psi(t)\right)
$$

Indeed, exploiting self-adjointness of $H$ one easily finds

$$
\begin{aligned}
& \frac{(\psi(t+h), H \psi(t+h))-(\psi(t), H \psi(t))}{h} \\
= & \left(\frac{\psi(t+h)-\psi(t)}{h}, H \psi(t+h)\right)+\left(H \psi(t), \frac{\psi(t+h)-\psi(t)}{h}\right)
\end{aligned}
$$


In order to prove (4.24) one needs only to show that

$$
\lim _{h \rightarrow 0} H \psi(t+h)=H \psi(t)
$$

in the strong $L^{2}$ sense. To this aim, one notices that, after some algebra, integrating by parts yields

$$
\begin{aligned}
& H \int_{t}^{t+h} d s e^{i H s}|\psi(s)|^{2} \psi(s) \\
= & -i e^{i H(t+h)}|\psi(t+h)|^{2} \psi(t+h)+i e^{i H t}|\psi(t)|^{2} \psi(t) \\
& +2 i \int_{t}^{t+h} d s e^{i H s}|\psi(s)|^{2} \partial_{t} \psi(s)+i \int_{t}^{t+h} d s e^{i H s} \psi^{2}(s) \partial_{t} \bar{\psi}(s)
\end{aligned}
$$

and taking the strong limit term by term one easily sees that the sum in the r.h.s. goes to zero. This proves (4.26) and thus, by (4.25), equality (4.24) is proven.

Then,

$$
\partial_{t}(\psi(t), H \psi(t))=-2 \lambda \operatorname{Im}\left(|\psi(t)|^{2} \psi(t), H \psi(t)\right)
$$

On the other hand,

$$
\partial_{t}\left(\psi(t),|\psi(t)|^{2} \psi(t)\right)=\partial_{t}\left(\psi^{2}(t), \psi^{2}(t)\right)=4 \lambda \operatorname{Im}\left(|\psi(t)|^{2} \psi(t), H \psi(t)\right)
$$

By (4.28) and (4.29) conservation of energy is proven.

Now we use the conservation laws to prove that strong solutions are global in time.

Proposition 4.5 (Eternity of strong solutions). Any solution to (1.2) with $\psi_{0} \in D(H)$ is global in $t$.

Proof. We treat the case of $H=H_{U}$ with $b \neq 0$ (see definition (2.3)). All other cases can be treated in the same way.

Using standard techniques one can prove that for any $\psi_{0}$ there exists a maximal time $T^{\star}\left(\psi_{0}\right)$ of existence for the solution $\psi(t)$ to the initial value problem $(4.20)$. We prove that $T^{\star}\left(\psi_{0}\right)$ is infinite. If not, by the blow-up alternative, one should have

$$
\lim _{t \rightarrow T^{\star}\left(\psi_{0}\right)}\|\psi(t)\|_{H}=+\infty
$$

On the other hand integration by parts yields

$$
\begin{aligned}
& (H+m) \int_{0}^{t} d s e^{-i H(t-s)}|\psi(s)|^{2} \psi(s) \\
= & -i|\psi(t)|^{2} \psi(t)+i e^{-i H t}\left|\psi_{0}\right|^{2} \psi_{0}+m \int_{0}^{t} d s e^{-i H(t-s)}|\psi(s)|^{2} \psi(s) \\
& +2 i \int_{0}^{t} d s e^{-i H(t-s)}|\psi(s)|^{2} \partial_{s} \psi(s)+i \int_{0}^{t} d s e^{-i H(t-s)} \psi^{2}(s) \partial_{s} \bar{\psi}(s)
\end{aligned}
$$

Then,

$$
\begin{aligned}
& \left\|\int_{0}^{t} d s e^{-i H(t-s)}|\psi(s)|^{2} \psi(s)\right\|_{H} \\
\leq & \|\psi(t)\|_{\infty}^{2}\|\psi(t)\|+\left\|\psi_{0}\right\|_{\infty}^{2}\left\|\psi_{0}\right\|+m \int_{0}^{t} d s\|\psi(s)\|_{\infty}^{2}\|\psi(s)\| \\
& +3 \int_{0}^{t} d s\|\psi(s)\|_{\infty}^{2}\|H \psi(s)\|+3|\lambda| \int_{0}^{t} d s\|\psi(s)\|_{\infty}^{2}\left\||\psi(s)|^{2} \psi(s)\right\|
\end{aligned}
$$


Using conservation of energy, conservation of $L^{2}$-norm, and inequality (3.15) first with $p=\infty$ and then with $p=4$ one easily finds that, for any $t<T^{\star}\left(\psi_{0}\right)$,

$$
\mathcal{E}\left[\psi_{0}\right]=\mathcal{E}[\psi(t)] \geq\left\|\phi^{\prime}(t)\right\|^{2}-2 M\left(\left\|\psi_{0}\right\|\right)\left\|\phi^{\prime}(t)\right\|
$$

where $\phi(t)$ is the regular part of $\psi(t)$ (see (2.19)) and

$$
M\left(\left\|\psi_{0}\right\|\right):=\frac{1}{2} C_{\infty}^{2}|b|^{-1}(|a|+|d|+2)\left\|\psi_{0}\right\|+\frac{|\lambda|}{4} C_{4}^{4}\left\|\psi_{0}\right\|^{3} .
$$

Therefore, from (4.33) one obtains

$$
\left\|\phi^{\prime}(t)\right\| \leq 2 M\left(\left\|\psi_{0}\right\|\right)+\sqrt{\mathcal{E}\left[\psi_{0}\right]}, \quad \text { for any } t<T^{\star}\left(\psi_{0}\right)
$$

and then, by (3.15) with $p=\infty$ one has

$$
\|\psi(t)\|_{\infty}^{2} \leq 2 C_{\infty}^{2}\left\|\psi_{0}\right\|\left[M\left(\left\|\psi_{0}\right\|\right)+\sqrt{\mathcal{E}\left[\psi_{0}\right]}\right]:=K\left(\psi_{0}\right), \quad \text { for any } t<T^{\star}\left(\psi_{0}\right) .
$$

From (4.32) one then has

$$
\begin{aligned}
& \left\|\int_{0}^{t} d s e^{-i H(t-s)}|\psi(s)|^{2} \psi(s)\right\|_{H} \\
\leq & K^{2}\left(\psi_{0}\right)\left[(2+m t)\left\|\psi_{0}\right\|+3 \int_{0}^{t} d s\|H \psi(s)\|+3|\lambda| \int_{0}^{t} d s\left\||\psi(s)|^{2} \psi(s)\right\|\right] \\
\leq & K^{2}\left(\psi_{0}\right)\left[\left(2+t\left(m+3|\lambda| K^{2}\left(\psi_{0}\right)\right)\right)\left\|\psi_{0}\right\|+3 \int_{0}^{t} d s\|\psi(s)\|_{H}\right] .
\end{aligned}
$$

Therefore,

$$
\|\psi(t)\|_{H} \leq\left\|\psi_{0}\right\|_{H}+|\lambda| K^{2}\left(\psi_{0}\right)\left[\left(2+t\left(m+3|\lambda| K^{2}\left(\psi_{0}\right)\right)\right)\left\|\psi_{0}\right\|+3 \int_{0}^{t} d s\|\psi(s)\|_{H}\right] .
$$

Then, by a Gronwall-type estimate one obtains

$$
\|\psi(t)\|_{H} \leq\left(\left\|\psi_{0}\right\|_{H}+2|\lambda| K^{2}\left(\psi_{0}\right)\left\|\psi_{0}\right\|+\frac{m}{3}\left\|\psi_{0}\right\|+|\lambda| K^{2}\left(\psi_{0}\right)\left\|\psi_{0}\right\|\right) e^{3|\lambda| K^{2}\left(\psi_{0}\right) t},
$$

then

$$
\limsup _{t \rightarrow T^{\star}\left(\psi_{0}\right)-0}\|\psi(t)\|_{H} \leq C\left(\psi_{0}\right) e^{3|\lambda| K^{2}\left(\psi_{0}\right) T^{\star}\left(\psi_{0}\right)}<+\infty
$$

that contradicts the blow-up alternative and then the finiteness of $T^{\star}\left(\psi_{0}\right)$. The proof is complete.

\section{Global well-posedness in $Q$}

In this section we prove the global well-posedness for the problem (1.2), provided that the operator $H$ is a s.a.e. of $\widehat{H}_{0}$ (see $(2.1),(2.2)$ ) with energy domain equal to $Q$ (see proposition 2.3). The other cases will be treated in the next section.

Lemma 5.1. For any function $\psi \in Q$,

$$
\left\||\psi|^{2} \psi\right\|_{Q} \leq C\|\psi\|_{Q}^{3} .
$$

Furthermore, for any couple of functions $\psi_{1}, \psi_{2} \in Q$ the following estimate holds:

$$
\left\|\left|\psi_{1}\right|^{2} \psi_{1}-\left|\psi_{2}\right|^{2} \psi_{2}\right\|_{Q} \leq C\left(\left\|\psi_{1}\right\|_{Q}^{2}+\left\|\psi_{2}\right\|_{Q}^{2}\right)\left\|\psi_{1}-\psi_{2}\right\|_{Q}
$$


Proof. By (3.16) $\psi$ is a bounded function, then $|\psi|^{2} \psi$ is in $L^{2}$. Exploiting decomposition $\psi=\phi+q \frac{\epsilon}{2}$ given in (2.19), we obtain an analogous decomposition for $|\psi|^{2} \psi$, namely

$$
|\psi|^{2} \psi=\eta+\mu \frac{\epsilon}{2}
$$

where

$$
\begin{aligned}
& \eta=|\phi|^{2} \phi+q^{2} \frac{\bar{\phi}}{4}+|q|^{2} \frac{\phi}{2}+\bar{q}\left[\phi^{2}-\phi^{2}(0)\right] \frac{\epsilon}{2}+2 q\left[|\phi|^{2}-|\phi(0)|^{2}\right] \frac{\epsilon}{2} \\
& \mu=\bar{q} \phi^{2}(0)+2 q|\phi(0)|^{2}+\frac{|q|^{2} q}{4}
\end{aligned}
$$

To show that $\eta^{\prime} \in L^{2}$ we first notice that $\eta$ is continuous, since the coefficient of $\epsilon$ appearing in the first equality in (5.3) vanishes at the origin. Now let us consider the first term in the definition (5.3) of $\eta$. It can be rewritten as $\phi^{2} \bar{\phi}$. Its $x$-derivative consists of two terms: we analyse only one of them, for instance $\phi^{2} \bar{\phi}^{\prime}$. To show that it belongs to $L^{2}$, we estimate the factor $\phi^{2}$ by its $L^{\infty}$-norm, so we are left with the $L^{2}$-norm of $\bar{\phi}^{\prime}$, which is finite. Then we obtain

$$
\left\|\phi^{2} \bar{\phi}^{\prime}\right\| \leq\|\phi\|_{\infty}^{2}\left\|\phi^{\prime}\right\| \leq C\|\psi\|_{Q}^{3} .
$$

The $L^{2}$-norm of the other terms in $\eta^{\prime}$ can be estimated similarly: indeed, each term consists of three factors, and it turns out that one of such factors is in $L^{2}$, while the two others can be estimated by their $L^{\infty}$-norm. Using remark 3.5 one finally obtains

$$
\left\||\psi|^{2} \psi\right\|_{Q} \leq C\|\psi\|_{Q}^{3} .
$$

Now we estimate the $Q$-norm of $\left|\psi_{1}\right|^{2} \psi_{1}-\left|\psi_{2}\right|^{2} \psi_{2}$. We have

$$
\left\|\left|\psi_{1}\right|^{2} \psi_{1}-\left|\psi_{2}\right|^{2} \psi_{2}\right\|_{Q} \leq||\left|\psi_{1}\right|^{2} \psi_{1}-\left|\psi_{2}\right|^{2} \psi_{2}\|+\| \eta_{1}^{\prime}-\eta_{2}^{\prime} \|
$$

where $\eta_{i}$ denotes the regular part of $\left|\psi_{i}\right|^{2} \psi_{i}$. For estimating the first term in the r.h.s of (5.5) we add and subtract the terms $\left|\psi_{1}\right|^{2} \psi_{2}$ and $\left|\psi_{2}\right|^{2} \psi_{1}$. By triangular inequality,

$$
\begin{aligned}
& \left\|\left|\psi_{1}\right|^{2} \psi_{1}-\left|\psi_{2}\right|^{2} \psi_{2}\right\| \leq\left(\left\|\psi_{1}\right\|_{\infty}^{2}+\left\|\psi_{1}\right\|_{\infty}\left\|\psi_{2}\right\|_{\infty}+\left\|\psi_{2}\right\|_{\infty}^{2}\right)\left\|\psi_{1}-\psi_{2}\right\| \\
\leq & C\left(\left\|\psi_{1}\right\|_{\infty}^{2}+\left\|\psi_{2}\right\|_{\infty}^{2}\right)\left\|\psi_{1}-\psi_{2}\right\|
\end{aligned}
$$

Now we estimate $\left\|\eta_{1}^{\prime}-\eta_{2}^{\prime}\right\|$. By formula (5.3) we have

$$
\begin{aligned}
& \left\|\eta_{1}^{\prime}-\eta_{2}^{\prime}\right\| \\
\leq & C\left(\left\|\left(\left|\phi_{1}\right|^{2} \phi_{1}\right)^{\prime}-\left(\left|\phi_{2}\right|^{2} \phi_{2}\right)^{\prime}\right\|+\left\|q_{1}^{2} \bar{\phi}_{1}^{\prime}-q_{2}^{2} \bar{\phi}_{2}^{\prime}\right\|+\left\|\left|q_{1}\right|^{2} \phi_{1}^{\prime}-\left|q_{2}\right|^{2} \phi_{2}^{\prime}\right\|\right. \\
& \left.+\left\|\bar{q}_{1}\left[\left(\phi_{1}^{2}-\phi_{1}^{2}(0)\right) \epsilon\right]^{\prime}-\bar{q}_{2}\left[\left(\phi_{2}^{2}-\phi_{2}^{2}(0)\right) \epsilon\right]^{\prime}\right\|+\left\|q_{1}\left[\left(\left|\phi_{1}\right|^{2}-\left|\phi_{1}(0)\right|^{2}\right) \epsilon\right]^{\prime}-q_{2}\left[\left(\left|\phi_{2}\right|^{2}-\left|\phi_{2}(0)\right|^{2}\right) \epsilon\right]^{\prime}\right\|\right) \\
= & (I)+(I I)+(I I I)+(I V)+(V)
\end{aligned}
$$

To estimate term $(I)$ we rewrite it as

$$
\begin{aligned}
& \left(\left|\phi_{1}\right|^{2} \phi_{1}\right)^{\prime}-\left(\left|\phi_{2}\right|^{2} \phi_{2}\right)^{\prime} \\
= & 2\left[\phi_{1} \phi_{1}^{\prime}\left(\bar{\phi}_{1}-\bar{\phi}_{2}\right)+\phi_{1} \bar{\phi}_{2}\left(\phi_{1}^{\prime}-\phi_{2}^{\prime}\right)+\phi_{2}^{\prime} \bar{\phi}_{2}\left(\phi_{1}-\phi_{2}\right)\right]+\bar{\phi}_{1}^{\prime}\left(\phi_{1}^{2}-\phi_{2}^{2}\right)+\phi_{2}^{2}\left(\bar{\phi}_{1}^{\prime}-\bar{\phi}_{2}^{\prime}\right)
\end{aligned}
$$


Then

$$
(I) \leq 2\left(\left\|\phi_{1}\right\|_{\infty}\left\|\phi_{1}^{\prime}\right\|+\left\|\phi_{2}\right\|_{\infty}\left\|\phi_{2}^{\prime}\right\|+\left\|\phi_{1}^{\prime}\right\|\left\|\phi_{1}+\phi_{2}\right\|_{\infty}\right)\left\|\phi_{1}-\phi_{2}\right\|_{\infty}+2\left(\left\|\phi_{1}\right\|_{\infty}^{2}+\left\|\phi_{2}\right\|_{\infty}^{2}\right)\left\|\phi_{1}^{\prime}-\phi_{2}^{\prime}\right\|
$$

Inequality (3.14) implies

$$
\left\|\phi_{1}-\phi_{2}\right\|_{\infty} \leq C\left\|\psi_{1}-\psi_{2}\right\|_{Q}
$$

Then, by (3.14), (5.9), (5.10)

$$
(I) \leq C\left(\left\|\psi_{1}\right\|_{Q}^{2}+\left\|\psi_{2}\right\|_{Q}^{2}\right)\left\|\psi_{1}-\psi_{2}\right\|_{Q}
$$

Terms $(I I),(I I I),(I V),(V)$ are estimated similarly using (3.13), (3.14) and (5.10), and the proof is complete.

Proposition 5.2 (Equivalence of energy norms). The energy space norms defined in (2.25) and (2.23) are equivalent.

Proof. We give the proof for the case at point 4. in the proposition (2.3). The others are simpler and can be treated the same way. So the energy space coincides with $Q$ and the expression of the form is given by (2.17).

Denoting by $\phi$ the regular part of $\psi$ and defining

$$
A:=\max \left(1, \frac{C_{\infty}^{2}|b|^{-2}(|a|+|d|+1)^{2}}{m}\right),
$$

where the constant $C_{\infty}$ was given in (3.15), one has

$$
\begin{aligned}
A\|\psi\|_{B}^{2} & \geq B(\psi)+C_{\infty}^{2}|b|^{-2}(|a|+|d|+1)^{2}\|\psi\|^{2} \\
& \geq\left\|\phi^{\prime}\right\|^{2}-C_{\infty}|b|^{-1}(|a|+|d|+1)\left\|\phi^{\prime}\right\|\|\psi\|+C_{\infty}^{2}|b|^{-2}(|a|+|d|+1)^{2}\|\psi\|^{2} \\
& \geq \frac{1}{2}\left(\left\|\phi^{\prime}\right\|^{2}+C_{\infty}^{2}|b|^{-2}(|a|+|d|+1)^{2}\|\psi\|^{2}\right) \geq c\|\psi\|_{Q}^{2},
\end{aligned}
$$

where $c:=\min \left(\frac{1}{2}, \frac{1}{2}|b|^{-2} C_{\infty}^{2}(|a|+|d|+1)^{2}\right)$.

On the other hand,

$$
\|\psi\|_{B}^{2} \leq\left\|\phi^{\prime}\right\|^{2}+C_{\infty}|b|^{-1}(|a|+|d|+1)\left\|\phi^{\prime}\right\|\|\psi\|+m\|\psi\|^{2} \leq C\|\psi\|_{Q}^{2}
$$

where $C:=\max (1, m)+\frac{1}{2}|b|^{-1} C_{\infty}(|a|+|d|+1)$.

So we showed the existence of $A, c, C>0$ s.t. $c A^{-1}\|\psi\|_{Q}^{2} \leq\|\psi\|_{B}^{2} \leq C\|\psi\|_{Q}^{2}$ and the proof is complete.

Lemma 5.3. Let $H$ be a s.a.e. of $\widehat{H}_{0}$ (see (2.1), (2.2)), and $X$ be the related energy space (see proposition 2.3). Then, for any $\psi_{0} \in X, t \in \mathbb{R}$,

$$
\left\|e^{-i t H} \psi_{0}\right\|_{X} \leq \tilde{C}\left\|\psi_{0}\right\|_{X}
$$

where the constant $\tilde{C}$ depends on $\psi_{0}$ and $H$, but not on $t$. 
Proof. As in the previous proof, we treat the case at point 4 . in proposition 2.3 only, the others being easier. Since $b \neq 0$, the domain of the form $B$ associated to the operator $H$ coincides with $Q$. Besides, the value of the form is conserved by the linear flow, namely

$$
B\left(\psi_{t}\right)=B\left(\psi_{0}\right)
$$

where we used the notation $\psi_{t}=e^{-i t H} \psi_{0}$. Then, from equation (2.21) and the unitary character of the propagator $e^{-i t H}$ in $L^{2}(\mathbb{R})$, denoting by $\phi_{t}$ the regular part of $\psi_{t}$ one has

$$
\left|B\left(\psi_{0}\right)\right| \geq\left\|\phi_{t}^{\prime}\right\|^{2}-C_{\infty}|b|^{-1}(|a|+|d|+1)\left\|\phi_{t}^{\prime}\right\|\left\|\psi_{0}\right\|
$$

where the constant $C_{\infty}$ was defined in (3.15).

From (5.15) one immediately has

$$
\left\|\phi_{t}^{\prime}\right\|^{2} \leq C\left|B\left(\psi_{0}\right)\right|
$$

where $C$ depends on $|a|,|b|,|d|$, and $\left\|\psi_{0}\right\|$ only.

Conversely,

$$
\left|B\left(\psi_{0}\right)\right| \leq\left\|\phi_{0}^{\prime}\right\|^{2}+|b|^{-1}(|a|+|d|+1)\left\|\phi_{0}^{\prime}\right\|\left\|\psi_{0}\right\| \leq C\left\|\psi_{0}\right\|_{Q}^{2}
$$

Then, from (5.17) and (5.15) one immediately has

$$
\left\|\phi_{t}^{\prime}\right\| \leq C\left\|\psi_{0}\right\|_{Q}
$$

and, since $\left\|\psi_{t}\right\|=\left\|\psi_{0}\right\|$, the proof is complete.

Now we can prove the well-posedness for the local Cauchy problem in the form domain of the s.a.e. $H$, provided that it coincides with the space $Q$.

Theorem 5.4 (Local existence and uniqueness for weak solutions in $Q$ ). Let $H$ be a s.a.e. of $\widehat{H}_{0}$ (see (2.1), (2.2)) whose corresponding quadratic form has domain $Q$.

Then, for any $\psi_{0} \in Q$ there exists $T \in(0,+\infty)$ such that the integral equation (1.2) has a unique solution $\psi \in C([0, T), Q)$.

Proof. Given $\psi_{0} \in Q$ we consider the Banach space

$$
\mathcal{Y}:=\left\{\psi \in Q,\|\psi\|_{Q} \leq 2 \tilde{C}\left\|\psi_{0}\right\|_{Q}\right\}
$$

where $\tilde{C}$ is the constant appearing in (5.14).

We define the operator $\Gamma$ acting on $L^{\infty}([0, T], \mathcal{Y})$, with $T$ to be specified:

$$
(\Gamma \psi)(t):=e^{-i t H} \psi_{0}-i \lambda \int_{0}^{t} d s e^{-i(t-s) H}|\psi(s)|^{2} \psi(s) .
$$

By lemmas 5.1, 5.3

$$
\|(\Gamma \psi)(t)\|_{Q} \leq \tilde{C}\left\|\psi_{0}\right\|_{Q}+C T\|\psi\|_{L^{\infty}([0, T], X)}^{3}
$$

and for any $\psi_{1}, \psi_{2} \in L^{\infty}([0, T], Q)$

$$
\left\|\left(\Gamma \psi_{1}\right)(t)-\left(\Gamma \psi_{2}\right)(t)\right\|_{Q} \leq C T\left(\left\|\psi_{1}\right\|_{L^{\infty}([0, T], Q)}^{2}+\left\|\psi_{2}\right\|_{L^{\infty}([0, T], Q)}^{2}\right)\left\|\psi_{1}-\psi_{2}\right\|_{L^{\infty}([0, T], Q)}
$$

From (5.19), (5.20) it follows that for $T=\left(9 C \tilde{C}^{2}\left\|\psi_{0}\right\|_{Q}^{2}\right)^{-1}, \Gamma$ is a contraction of $L^{\infty}([0, T], \mathcal{Y})$, then there exists a unique solution of equation $(1.2)$ in $L^{\infty}([0, T], \mathcal{Y})$.

By a one-step bootstrap in (1.2) it is immediately seen that the solution actually belongs to $C^{0}([0, T], \mathcal{Y})$. 
Now we give a simple lemma in order to immediately prove a corollary on well-posedness. Yet the lemma proves useful for showing the conservation laws too.

Lemma 5.5. The function $t \mapsto e^{i H t} \psi(t)$ is differentiable both as a function in $L^{2}$ and in $Q$, and

$$
\partial_{t} e^{i H t} \psi(t)=-i \lambda e^{i H t}|\psi(t)|^{2} \psi(t)
$$

Proof. Indeed,

$$
\begin{aligned}
& |\lambda|\left\|h^{-1} \int_{t}^{t+h} e^{i H s}|\psi(s)|^{2} \psi(s) d s-e^{i H t}|\psi(t)|^{2} \psi(t)\right\| \\
\leq & |\lambda||h|^{-1} \int_{t}^{t+h} d s\left\|e^{i H s}|\psi(s)|^{2} \psi(s)-e^{i H t}|\psi(t)|^{2} \psi(t)\right\| \\
\leq & |\lambda||h|^{-1} \int_{0}^{h} d s\left\|e^{i H(t+s)}|\psi(t+s)|^{2} \psi(t+s)-e^{i H(t+s)}|\psi(t)|^{2} \psi(t)\right\| \\
& +|\lambda||h|^{-1} \int_{0}^{h} d s\left\|e^{i H(t+s)}|\psi(t)|^{2} \psi(t)-e^{i H t}|\psi(t)|^{2} \psi(t)\right\|
\end{aligned}
$$

The first term can be estimated using continuity in time of the solution $\psi$, the second by the continuity of the propagator $e^{-i H t}$. Notice that the norm can be understood either in $L^{2}$ or in $Q$.

Corollary 5.6. The local solution in $Q$ to the problem (1.2) lies in the space $C([0, T], Q) \cap C^{1}([0, T), Q)$.

Proof. Consider a function $\xi \in D(H)$. One then has

$$
\begin{aligned}
\left\langle\partial_{t} \psi(t), \xi\right\rangle_{Q^{\star}, Q}: & =\partial_{t}(\psi(t), \xi)=\partial_{t}\left(e^{i H t} \psi(t), e^{i H t} \xi\right) \\
& =\left(-i \lambda|\psi(t)|^{2} \psi(t), \xi\right)+B(\psi(t), \xi)
\end{aligned}
$$

where we used formula (5.21).

Then

$$
\left|\left\langle\partial_{t} \psi(t), \xi\right\rangle_{Q^{\star}, Q}\right| \leq|\lambda|\|\psi(t)\|_{Q}^{3}\|\xi\|+C\|\psi(t)\|_{Q}\|\xi\|_{Q},
$$

so $\partial_{t} \psi(t)$ belongs to $Q^{\star}$. Furthermore, the correspondence $t \mapsto \partial_{t} \psi(t)$ is continuous in $Q^{\star}$ due to the continuity of $t \mapsto \psi(t)$ in $Q$ and to estimate (5.24).

Remark 5.7 (Blow-up alternative). Since the size $T$ of the time interval where the solution exists depends on the $Q$-norm of the solution only, one obtains the following alternative: either the solution is global in time, or there exists $T^{\star}<\infty$ such that

$$
\lim _{t \rightarrow T^{\star}-0}\|\psi(t)\|_{Q}=+\infty .
$$

\subsection{Conservation laws and global existence}

As for the usual nonlinear Schrödinger equation, the main conserved quantities are the $L^{2}$-norm and the energy of the solution.

Proposition 5.8 (Conservation of the $L^{2}$-norm). For any solution $\psi \in C([0, T), Q)$ to the problem (1.2), the following conservation law holds:

$$
\|\psi(t)\|=\left\|\psi_{0}\right\| \quad \text { for any } t \in[0, T) .
$$


Proof. Using (5.21) one immediately finds

$$
\begin{aligned}
\partial_{t}(\psi(t), \psi(t)) & =\partial_{t}\left(e^{i H t} \psi(t), e^{i H t} \psi(t)\right)=2 \operatorname{Re}\left(e^{i H t} \psi(t), \partial_{t} e^{i H t} \psi(t)\right) \\
& =2 \operatorname{Im}\left(e^{i H t} \psi(t), e^{i H t}|\psi(t)|^{2} \psi(t)\right)=2 \operatorname{Im}\left(\psi(t),|\psi(t)|^{2} \psi(t)\right)=0
\end{aligned}
$$

Proposition 5.9 (Conservation of the energy for solutions in $Q$ ). For any solution $\psi \in C([0, T), Q)$ to the problem (1.2) the conservation of energy (4.23) holds.

Proof. Let $T^{\star}$ be the maximal time of existence of $\psi$. Due to the density of $D(H)$ in $Q$, there exists a sequence $\psi_{0, n} \in D(H)$ that converges to $\psi_{0}$ in $Q$. We prove that time evolution propagates the convergence in $Q$ at any time $T \in\left(0, T^{\star}\right)$.

Indeed, denoted by $\psi_{n}(t)$ the solution to (1.2) with initial data $\psi_{0, n}$, one immediately has

$$
\psi(t)-\psi_{n}(t)=e^{-i H t}\left(\psi_{0}-\psi_{0, n}\right)-i \lambda \int_{0}^{t} d s e^{-i H(t-s)}\left(|\psi(s)|^{2} \psi(s)-\left|\psi_{n}(s)\right|^{2} \psi_{n}(s)\right)
$$

Then, using (5.14) and (5.2) we have

$$
\left\|\psi(t)-\psi_{n}(t)\right\|_{Q} \leq C\left\|\psi_{0}-\psi_{0, n}\right\|_{Q}+C \int_{0}^{t} d s\left(\|\psi(s)\|_{Q}^{2}+\left\|\psi_{n}(s)\right\|_{Q}^{2}\right)\left\|\psi(s)-\psi_{n}(s)\right\|_{Q}
$$

From (4.35) we know that $\left\|\psi_{n}(s)\right\|_{Q}$ is bounded uniformly in $s$ and $n$. Notice that $\|\psi(s)\|_{Q}$ must be bounded for $s$ in $(0, T)$ too, otherwise the blow-up alternative would prevent the solution from being extended up to $T^{\star}$. Therefore, there exists $C_{T}>0$ such that, by (5.28),

$$
\left\|\psi(t)-\psi_{n}(t)\right\|_{Q} \leq C\left\|\psi_{0}-\psi_{0, n}\right\|_{Q}+C_{T} \int_{0}^{t} d s\left\|\psi(s)-\psi_{n}(s)\right\|_{Q}
$$

and then, by standard Gronwall's inequality,

$$
\left\|\psi(t)-\psi_{n}(t)\right\|_{Q} \leq C e^{C_{T} T}\left\|\psi_{0}-\psi_{0, n}\right\|_{Q}, \quad \forall t \in[0, T)
$$

Then, we have that

$$
\lim _{n \rightarrow \infty} \psi_{n}(t)=\psi(t), \quad \text { in the topology of } Q, \text { for any } t \in[0, T) .
$$

Since the energy functional is continuous in $Q$, and exploiting conservation of the energy for solutions in $H$, we get

$$
\mathcal{E}[\psi(t)]=\lim _{n \rightarrow \infty} \mathcal{E}\left[\psi_{n}(t)\right]=\lim _{n \rightarrow \infty} \mathcal{E}\left[\psi_{0, n}\right]=\mathcal{E}\left[\psi_{0}\right]
$$

and the proposition is proven.

Proposition 5.10 (Eternity of solutions in Q). Any solution of (1.2) with $\psi_{0} \in Q$ is global in t.

Proof. It suffices to proceed further with the previous proof. The energy conservation (5.32) implies inequality (4.35). Therefore, the quantity $\|\psi(t)\|_{Q}$ can be estimated uniformly in $t \in\left(0, T^{\star}\right)$. Therefore, by continuity of time evolution in $Q,\left\|\psi\left(T^{\star}\right)\right\|_{Q}$ is bounded, and so the blow-up alternative implies $T^{\star}=+\infty$.

Remark 5.11. All proofs in this section can be extended to any s.a.e. $H$ of $\widehat{H}_{0}$ whose energy domain is $Q_{0}, Q_{0+}, Q_{0-}$ or $Q_{ \pm \omega}$ : the only condition is the stability of the energy domain under the action of the cubic nonlinearity. 


\section{Global well-posedness in $Q_{\omega a}$}

In this section we prove the well-posedness for the problem (1.2) in the form domain $Q_{\omega a}$ of a hamiltonian operator $H$ defined by boundary conditions $(2.3)$ with $b=0$.

When dealing with such s.a. extensions the main problem lies in the fact that the boundary condition that defines $Q_{\omega a}$ is not stable under the action of the nonlinearity, therefore the simple proof given for the well-posedness in $Q$ cannot be extended to this case and a more careful analysis is required.

As already pointed out, if $a= \pm 1$ then the proof for the problem in $Q$ holds for this case too.

In order to cover all other cases we shall use the "integrated by parts" form of the Duhamel formula. To that purpose we need to extend the action of the resolvent of $H$ from $L^{2}(\mathbb{R})$ to the whole space $Q^{\star}$.

\subsection{Extension of the resolvent to $Q^{\star}$}

We start by extending the resolvent of the free Laplacian to $H^{-1}(\mathbb{R})$.

Definition 6.1. Let $H_{0}$ be the s.a.e. of $\widehat{H}_{0}$ (see (2.1), (2.2)), defined on $H^{2}(\mathbb{R})$. For any $z \in$ $\mathbb{C} \backslash(-\infty, 0]$ denote by $R_{0}(z)$ the resolvent operator $\left(H_{0}+z\right)^{-1}$, acting on $L^{2}(\mathbb{R})$.

We define the extended free resolvent $\widetilde{R}_{0}(z)$ as follows. Given $f \in Q^{\star}$,

$$
\widetilde{R}_{0}(z) f=\mathcal{F}^{-1} \frac{\widehat{f}_{1}(k)}{k^{2}+z}
$$

where, according to (2.40), $f_{1}$ is the $H^{-1}$-component of $f, \widehat{f_{1}}$ is its Fourier transform as a Schwartz distribution, and $\mathcal{F}^{-1}$ denotes the inverse Fourier transform in the same space.

Remark 6.2. We point out that:

- for any $f \in Q^{\star}, \widetilde{R}_{0}(z) f \in H^{1}(\mathbb{R})$;

- $\widetilde{R}_{0}(z)$ is not invertible, indeed its kernel coincides with the subspace of $Q^{\star}$ generated by $\delta(0+)-$ $\delta(0-)$. However, its restriction on $H^{-1}(\mathbb{R})$ is invertible;

- $\widetilde{R}_{0}(z)$ is bounded as an operator from $Q^{\star}$ to $H^{1}(\mathbb{R})$.

Indeed,

$$
\left\|\widetilde{R}_{0}(z) f\right\|_{H^{1}(\mathbb{R})}^{2} \leq C \int_{\mathbb{R}} \frac{\left|\widehat{f}_{1}(k)\right|^{2}}{k^{2}+1} d k \leq C\|f\|_{Q^{\star}}^{2}
$$

As a second step we extend to $Q^{\star}$ the resolvent of the s.a.e. of $\widehat{H}_{0}$ with Dirichlet boundary condition at zero.

Definition 6.3. Let $H_{0,0}$ be the s.a.e. of $\widehat{H}_{0}$ (see (2.1), (2.2)), whose domain $D_{D}$ contains all functions in $H^{2}(\mathbb{R})$ vanishing at $x=0$. For any $z \in \mathbb{C} \backslash(-\infty, 0]$ denote by $R_{D}(z)$ the resolvent operator $\left(H_{0,0}+z\right)^{-1}$ acting on $L^{2}(\mathbb{R})$.

We define the action of the extended resolvent $\widetilde{R}_{D}(z)$ on $f \in Q^{\star}$ as follows:

$$
\widetilde{R}_{D}(z) f:=\widetilde{R}_{0}(z) f-\frac{\varphi^{z}}{2 \sqrt{z}}\left\langle f, \varphi^{z}\right\rangle_{Q^{\star}, Q}
$$

where the functions $\varphi^{z}$ were introduced in section 1.1 . 
Remark 6.4. Notice that:

- $\widetilde{R}_{D}(z) f=R_{D}(z) f$ if $f \in L^{2}(\mathbb{R})$.

- $\widetilde{R}_{D}(z)$ is a continuous linear map from $Q^{\star}$ to $H_{0}^{1}(\mathbb{R})$.

Indeed, from continuity of $\widetilde{R}_{0}(z)$ and continuity of the second term in the definition (6.3) of $\widetilde{R}_{0}(z)$, we have that $\widetilde{R}_{D}(z)$ is continuous from $Q^{\star}$ to $H^{1}(\mathbb{R})$. Now, fix $f \in Q^{\star}$ and consider a sequence $f_{n} \in L^{2}(\mathbb{R}) \cap Q^{\star}$ that converges to $f$ in the topology of $Q^{\star}$. Obviously, $\widetilde{R}_{D} f_{n}(0)=0$, and by continuity $\widetilde{R}_{D} f_{n}$ converges to $\widetilde{R}_{D} f$ in $H^{1}(\mathbb{R})$. But this implies pointwise convergence, then $\widetilde{R}_{D} f(0)=0$.

- Decomposing $f$ as in (2.34) one immediately has

$$
\widetilde{R}_{D}(z) f=\widetilde{R}_{0}(z) f_{0}-\frac{\varphi^{z}}{2 \sqrt{z}}\left\langle f_{0}, \varphi^{z}\right\rangle_{Q^{\star}, Q}
$$

- From (6.4),

$$
\widetilde{R}_{D}(z) f=\widetilde{R}_{D}(z) f_{0}
$$

Now we can extend to $Q^{\star}$ the resolvent of any s.a.e. of $\widehat{H}_{0}$.

Definition 6.5. Let $H$ be any s.a.e. of $\widehat{H}_{0}$. For any $z$ in the resolvent set of $H$ we define the extended resolvent $\widetilde{R}(z)$ as follows:

$$
\widetilde{R}(z) f:=\widetilde{R}_{D}(z) f+\sum_{j, k= \pm} \frac{\mu_{j, k}(z)}{2 \sqrt{z}} \varphi_{j}^{z}\left\langle f, \varphi_{k}^{z}\right\rangle_{Q^{\star}, Q}
$$

where the coefficients $\alpha$ and $\beta$ were defined in (2.34), the function $\varphi_{j}^{z}$ in (1.4), and the parameters $\mu_{j, k}(z)$ give the difference between the ordinary resolvent operators $R(z)-R_{D}(z)$ according to Krein's formula (see [3], chapter 7, section 84).

Remark 6.6. As in the previously discussed cases,

- $\widetilde{R}(z) f=(H+z)^{-1}(z) f$ if $f \in L^{2}(\mathbb{R})$.

- it is easily seen that $\widetilde{R}(z)$ is a continuous linear map from $Q^{\star}$ to $Q$;

- by decomposition (2.34), one has

$$
\widetilde{R}(z) f:=\widetilde{R}_{D}(z) f_{0}+\sum_{j, k= \pm} \frac{\mu_{j, k}(z)}{2 \sqrt{z}} \varphi_{j}^{z}\left\langle f_{0}, \varphi_{k}^{z}-\varphi_{k}\right\rangle_{Q^{\star}, Q}+\frac{\alpha}{2 \sqrt{z}} \sum_{j= \pm} \mu_{j,+}(z) \varphi_{j}^{z}+\frac{\beta}{2 \sqrt{z}} \sum_{j= \pm} \mu_{j,-}(z) \varphi_{j}^{z}
$$

Remark 6.7. Applying Krein's theory one easily verifies that, if $H$ is a hamiltonian operator defined by the boundary condition (2.3) with $b=0, a \neq \pm 1$, then the action of its extended resolvent is represented by the integral kernel

$$
\begin{aligned}
\widetilde{R}_{\omega, a, c}(z ; x, y) f:= & \widetilde{R}_{D}(z ; x, y) f+\frac{1}{\left(a^{2}+1\right) \sqrt{z}+a c}\left[a^{2} \theta_{+}(x) \theta_{+}(y) e^{-\sqrt{z} x} e^{-\sqrt{z} y}\right. \\
& \left.+\omega a \theta_{+}(x) \theta_{-}(y) e^{-\sqrt{z} x} e^{\sqrt{z} y}+\bar{\omega} a \theta_{-}(x) \theta_{+}(y) e^{\sqrt{z} x} e^{-\sqrt{z} y}+\theta_{-}(x) \theta_{-}(y) e^{\sqrt{z} x} e^{\sqrt{z} y}\right]
\end{aligned}
$$

where the functions $\varphi_{ \pm}^{z}$ were explicitly written.

Moreover, 
- for any $f \in Q^{\star}$, we find $\widetilde{R}_{\omega, a, c}(z) f \in Q_{\omega a}$. To prove it, we just notice that for any $f \in Q^{\star}$

$$
\begin{aligned}
& \widetilde{R}_{\omega, a, c}(z) f(0+)=\frac{1}{\left(a^{2}+1\right) \sqrt{z}+a c}\left[a^{2}\left\langle f, \varphi_{+}^{z}\right\rangle_{Q^{\star}, Q}+\omega a\left\langle f, \varphi_{-}^{z}\right\rangle_{Q^{\star}, Q}\right] \\
& \widetilde{R}_{\omega, a, c}(z) f(0-)=\frac{1}{\left(a^{2}+1\right) \sqrt{z}+a c}\left[\bar{\omega} a\left\langle f, \varphi_{+}^{z}\right\rangle_{Q^{\star}, Q}+\left\langle f, \varphi_{-}^{z}\right\rangle_{Q^{\star}, Q}\right] .
\end{aligned}
$$

Then the correct boundary condition is fulfilled;

- $\widetilde{R}_{\omega, a, c}(z)$ is not invertible, since its kernel coincides with the subspace of $Q^{\star}$ generated by $\delta(0+)-\omega a \delta(0-)$. However, its restriction to $Q_{\omega a}^{\star}$ is invertible;

- $\widetilde{R}_{\omega, a, c}(z)$ is bounded as an operator from $Q^{\star}$ to $Q_{\omega a}$.

\subsection{Proof of the well-posedeness}

Before proving the well-posedness for the problem (1.2) in the space $Q_{\omega a}^{\star}$, we notice that, by using continuity of $\widetilde{R}(m)$ one immediately has the following lemma:

Lemma 6.8. Let $f$ be a map in $C^{0}\left([0, T), Q_{\gamma}\right) \cap C^{1}\left([0, T), Q_{\rho}^{\star}\right)$, with $\gamma, \rho \in \mathbb{C}$. Then,

$$
\partial_{t} e^{i(H+m) t} \widetilde{R}(m) f(t)=i e^{i(H+m) t} f(t)+e^{i(H+m) t} \widetilde{R}(m) \partial_{t} f(t),
$$

where $H$ is any s.a.e. of $\widehat{H}_{0}$ and the derivative of $f$ is to be understood in $Q_{\rho}^{\star}$.

Then formula (4.6) can be generalized to

$$
\begin{aligned}
& \int_{0}^{t} d s e^{-i H(t-s)}|\psi(s)|^{2} \psi(s) \\
= & -i \widetilde{R}(m)|\psi(t)|^{2} \psi(t)+i e^{-i H t} \widetilde{R}(m)\left|\psi_{0}\right|^{2} \psi_{0}+m \widetilde{R}(m) \int_{0}^{t} d s e^{-i H(t-s)}|\psi(s)|^{2} \psi(s) \\
& +2 i \widetilde{R}(m) \int_{0}^{t} d s e^{-i H(t-s)}|\psi(s)|^{2} \partial_{s} \psi(s)+i \widetilde{R}(m) \int_{0}^{t} d s e^{-i H(t-s)} \psi^{2}(s) \partial_{s} \bar{\psi}(s) .
\end{aligned}
$$

We finally prove global well-posedness in $Q_{\omega a}$.

Theorem 6.9 (Existence and uniqueness for global solutions in $\left.Q_{\omega a}\right)$. Let $H$ be any self-adjoint extension of the operator $\widehat{H}_{0}$ (see (2.1), (2.2)), defined by the boundary conditions (2.3) with $b=0$, $a \neq \pm 1$. Let its energy domain be denoted by $Q_{\omega a}$.

Then, for any $\psi_{0} \in Q_{\omega a}$ the equation (1.2) has a unique solution $\psi \in C\left([0,+\infty), Q_{\omega a}\right) \cap$ $C^{1}\left([0,+\infty), Q_{\omega a}^{\star}\right)$. Moreover, for the solution the conservation laws of $L^{2}$-norm and of energy $B_{\omega a}(\psi(t))+\lambda / 2\|\psi(t)\|_{4}^{4}$ hold.

Proof. We define $\mathcal{Z}:=C\left([0, T), Q_{\omega a}\right) \cap C^{1}\left([0, T), Q_{\omega a}^{\star}\right)$ and show that the map

$$
\begin{aligned}
\Theta & : \mathcal{Z}_{r} \rightarrow \mathcal{Z}_{r} \\
& \psi \mapsto e^{-i H \cdot} \psi_{0}-i \lambda \int_{0}^{\cdot} d s e^{i H(\cdot-s)}|\psi(s)|^{2} \psi(s),
\end{aligned}
$$

where $\mathcal{Z}_{r}$ is a closed ball of radius $r$ (to be chosen) in $\mathcal{Z}$, is a contraction, at least for small values of $T$. 
The proof of the first estimate of interest closely follows the line of the proof of the bound (4.8) in theorem 4.1. Some more care is required in the estimate of the two last term of (6.10). We show how to proceed considering the second-last term.

By continuity of $\widetilde{R}(m): Q_{\omega a^{-1}}^{\star} \rightarrow Q_{\omega a}$ one gets

$$
\left\|\widetilde{R}(m)|\psi(t)|^{2} \partial_{t} \psi(t)\right\|_{Q_{\omega a}} \leq C|||\psi(t)|^{2} \partial_{t} \psi(t) \|_{Q_{\omega a}^{\star}} .
$$

Furthermore, approximating $\partial_{t} \psi(t)$ by a sequence in $L^{2}$ and using continuity it is clear that the multiplication by $|\psi(t)|^{2}$ is to be understood by duality, therefore

$$
\left\langle|\psi(t)|^{2} \partial_{t} \psi(t), \xi\right\rangle_{Q_{\omega a^{-1}}^{\star}, Q_{\omega a^{-1}}}=\left\langle\partial_{t} \psi(t),|\psi(t)|^{2} \xi\right\rangle_{Q_{\omega a}^{\star}, Q_{\omega a}} .
$$

where $\xi$ is an element of $Q_{\omega a^{-1}}$.

Hence,

$$
\left\||\psi(t)|^{2} \partial_{t} \psi(t)\right\|_{Q_{\omega a}^{\star}-1} \leq\|\psi(t)\|_{\infty}^{2}\left\|\partial_{t} \psi(t)\right\|_{Q_{\omega a}^{\star}} \leq C\|\psi(t)\|_{Q_{\omega a}}^{2}\left\|\partial_{t} \psi(t)\right\|_{Q_{\omega a}^{\star}}
$$

Then,

$$
\left\|\widetilde{R}(m)|\psi(t)|^{2} \partial_{t} \psi(t)\right\|_{Q_{\omega a}} \leq C\|\psi(t)\|_{Q_{\omega a}}^{2}\left\|\partial_{t} \psi(t)\right\|_{Q_{\omega a}^{\star}}
$$

Now we estimate the time derivative of $\Theta \psi(t)$ as a functional on $Q_{\omega a}^{\star}$. First, we define it in the usual way: given $\zeta \in Q_{\omega a}$

$$
\langle\Theta \psi(t), \zeta\rangle_{Q_{\omega a}^{\star}, Q_{\omega a}}:=(\Theta \psi(t), \zeta) .
$$

Then,

$$
\begin{aligned}
\left\langle\partial_{t} \Theta \psi(t), \zeta\right\rangle_{Q_{\omega a}^{\star}, Q_{\omega a}} & :=\partial_{t}(\Theta \psi(t), \zeta)=i \lambda\left(|\psi(t)|^{2} \psi(t), \zeta\right)+B_{\omega a}(\psi(t), \zeta) \\
& \leq C\|\psi(t)\|_{Q_{\omega a}}^{3}\|\zeta\|_{Q_{\omega a}}
\end{aligned}
$$

where we exploited formula (5.21), with $e^{i H t} \Theta \psi(t)$ replacing $e^{i H t} \psi(t)$ in the l.h.s.. The proof proceeds as in theorem 4.1 so we omit repeating it.

We can conclude

$$
\|\Theta \psi\|_{\mathcal{Z}} \leq \tilde{C}\left\|\psi_{0}\right\|_{Q_{\omega a}}+C T\|\psi\|_{\mathcal{Z}}
$$

For the proof of the Lipschitz condition we first consider the $C\left([0, T), Q_{\omega a}\right)$ norm. The situation is analogous to the one discussed in the proof of the analogous point in theorem (4.1). More care is needed to treat the analogues of terms $(I X)$ and $(X)$ in that proof. We show how to proceed for term analogous to $(I X)$, the other being very akin.

$\left\||\psi(t)|^{2} \partial_{t} \psi(t)-|\xi(t)|^{2} \partial_{t} \xi(t)\right\|_{Q_{\omega a}^{\star}-1} \leq\left\||\psi(t)|^{2}-|\xi(t)|^{2}\right\|_{Q}\left\|\partial_{t} \psi(t)\right\|_{Q_{\omega a}^{\star}}+\|\xi(t)\|_{Q_{\omega \alpha}}^{2}\left\|\partial_{t} \psi(t)-\partial \xi(t)\right\|_{Q_{\omega a}^{\star}}$ and then

$$
\begin{aligned}
& \|\Theta \psi-\Theta \xi\|_{C\left([0, T), Q_{\omega a}\right)}=|\lambda|\left\|\widetilde{R}(m) \int_{0}^{\cdot} d s e^{-i H(\cdot-s)}\left(|\psi(s)|^{2} \partial_{s} \psi(s)-|\xi(s)|^{2} \partial_{s} \xi(s)\right)\right\|_{C\left([0, T), Q_{\omega a}\right)} \\
& \leq C T\left(\|\psi\|_{\mathcal{Z}}^{2}+\|\xi\|_{\mathcal{Z}}^{2}\right)\|\psi-\xi\|_{\mathcal{Z}}
\end{aligned}
$$

The Lipschitz bound in the norm $C^{1}([0, T), \mathcal{Z})$ is easily obtained applying formula (6.16). So we have

$$
\|\Theta \psi-\Theta \xi\|_{\mathcal{Z}} \leq C T\left(\|\psi\|_{\mathcal{Z}}^{2}+\|\xi\|_{\mathcal{Z}}^{2}\right)\|\psi-\xi\|_{\mathcal{Z}}
$$


Mimicking the proof of theorem 4.1 from formula (4.17) to the end we prove that $\Theta$ is a contraction when restricted to a suitable ball centered at the origin and a suitable time interval $[0, T / 2]$. Since both the size of the ball and $T$ depend on $\left\|\psi_{0}\right\|_{Q_{\omega a}}$ only, we have the blow-up alternative.

Conservation of the $L^{2}$-norm and of the energy, and therefore the global character of the solution, can be proved following the line used for the analogous issues for solutions in $Q$. The only delicate point arises when proving the conservation of the energy. Indeed, the persistence of a boundary condition in the definition of the energy domain prevents one from directly generalizing (5.30) to $Q_{\omega a}$. We sketch the necessary modifications to the proof of proposition 5.9: first, as in the case of $Q$, one approximates the initial data $\psi_{0}$ by a sequence $\psi_{0, n}$ in $D(H)$; second, denoted by $\psi_{n}$ the solution to (1.2) with initial data $\psi_{0, n}$, and using that $\psi(t)$ lies in $Q_{\omega a}$, one proves, proceeding as in proposition 5.9 , that

$$
\left\|\psi(t)-\psi_{n}(t)\right\| \leq C e^{C_{T} T}\left\|\psi_{0}-\psi_{0, n}\right\|, \quad \forall t \in[0, T)
$$

for any $T$ in the existence interval of $\psi$. Therefore $\psi_{n}(t)$ converges to $\psi(t)$ uniformly in $L^{2}(\mathbb{R})$; third, integrating by parts in the Duhamel's formula (see (6.10)) one gets

$$
\left\|\psi_{n}(t)-\psi(t)\right\|_{Q_{\omega a}} \leq C\left(\left\|\psi_{0, n}-\psi_{0}\right\|_{Q_{\omega a}}+\max _{t \in[0, T]}\left\|\psi_{n}(t)-\psi_{0}(t)\right\|\right)+C \int_{0}^{t} d s\left\|\partial_{s} \psi_{n}(s)-\partial_{s} \psi(s)\right\|_{Q_{\omega a}^{\star}} .
$$

Finally, using (6.20) and Gronwall's inequality again one has that $\psi_{n}(t)$ converges to $\psi(t)$ in $Q_{\omega a}$. Then, by continuity of energy in $Q_{\omega a}$, the proof is complete.

Remark 6.10. The well-posedness result holds in the space $C\left([0,+\infty), Q_{\omega a}\right)$ too. Indeed, from (5.23), (5.24) we immediately obtain that any solution in such space has a time derivative in $Q_{\omega a}^{\star}$.

\section{Acknowledgements.}

This research was partially supported by a "Borsa per giovani ricercatori GNFM 2008".

We are grateful to Prof. Andrea Sacchetti for many valuable discussions and suggestions, and to Dr. Alberto Ferrero for a useful discussion concerning duals of energy spaces.

During the final draft of this paper R.A. was visiting the Department of Mathematics of the University of California at Berkeley, which he thanks for the kind hospitality.

\section{References}

[1] Adami R., Golse, F, Teta A.: Rigorous derivation of the cubic NLS in dimension one, J. Stat. Phys. 127 1193-1220 (2007)

[2] Adami R., Sacchetti A.: The transition from diffusion to blow-up for a NLS equation in dimension one, J.Phys. A 38 8379-8392 (2005)

[3] Akhiezer N., Glazman I.: Theory of linear operators in Hilbert spaces, New York, Ungar, (1963).

[4] Albeverio S., Brzeźniak Z., Dabrowski L.: Fundamental solutions of the Heat and Schrödinger Equations with point interaction, Journal of Functional Analysis, 130 (1995) 220-254.

[5] Albeverio S., Gesztesy F., Hoegh-Krohn R., Holden H.: Solvable Models in Quantum Mechanics. New York: Springer-Verlag 1988 
[6] Albeverio S., Kurasov P.: Singular Perturbations of Differential Operators, Cambridge University Press 2000

[7] Bergé L.: Wave collapse in physics: principles and applications to light and plasma waves, Phys.Rep. 303 259-370 (1999)

[8] Brazhnyi V., Konotop V., Perez-García V.: Driving defect modes of Bose Einstein condensates in optical lattices, Phys. Rev.Lett. 96060403 (2006)

[9] Brazhnyi V., Konotop V., Perez-García V.: Defect modes of a Bose Einstein condensate in an optical lattice with a localized impurity, Phys. Rev.A, 74023614 (2006).

[10] Cao Xiang D., Malomed A. B.: Soliton defect collisions in the nonlinear Schrödinger equation, Phys. Lett. A 206 177-182 (1995)

[11] Cazenave, T.: Semilinear Schrödinger Equations, vol. 10 Courant Lecture Notes in Mathematics $A M S$, Providence (2003).

[12] Coclite, G. M., Holden, H.: The Schrödinger-Maxwell system with Dirac mass, Ann.Inst. H. Poincaré, Anal. Non Lin. 24, 773-793, (2007) and Erratum, 25, 833-866, (2008).

[13] D'Ancona P. Pierfelice V. Teta A.: Dispersive estimates for the Schrödinger equation with point interactions, Mathematical Methods in the Applied Sciences, 29 (2006), 309-323

[14] Della Casa F. Sacchetti A.: Stationary states for nonlinear one dimensional Schrödinger equation with singular potential, Physica D 209 60-68 (2006)

[15] Exner P., Grosse P.: Some properties of the one-dimensional generalized point interactions (a torso), mp-arc 99-390, math-ph/9910029 (1999).

[16] Erdős L., Schlein B., Yau H-T.: Derivation of the cubic nonlinear Schrödinger equation from quantum dynamics of many body systems, Invent. Math. 167 515-614, (2007)

[17] Erdős L., Schlein B., Yau H-T.: Derivation of the Gross-Pitaevskii equation for the dynamics of the Bose Einstein condensate., Ann. Math., to appear, (2006)

[18] Fukuizumi R., Ohta M, Ozawa T.: Nonlinear Schrödinger equation with a point defect, Ann. I.H.Poincaré, AN, 25 (2008), 837-845

[19] Goldstein J. A.: Semigroups of Linear Operators and Applications. Oxford Univ. Press 1985

[20] Goodman R. H., Holmes P. J., Weinstein M. I.: Strong NLS soliton-defect interactions Physica D, 192 (2004), 215-248

[21] Holmer J., Marzuola J., Zworski M.: Fast soliton scattering by delta impurities, Comm. Math. Phys, 274 (2007), 187-216.

[22] Kuchment, P.: Quantum graphs: I. Some basic structures, Waves in Random and Complex Media, 14, 1, (2003), S107-S128.

[23] Lieb E. H., Seiringer R., Solovej J.-P., Yngvason J.: The mathematics of the Bose gas and its condensation, Oberwolfach Seminar, 2005. 
[24] Sandoval Romero M., Weder R.: The initial value problem, scattering and inverse scattering, for Schrödinger equations with a potential and a non-local nonlinearity, J. Phys. A, 39, 37 (2006), 11461-11478.

[25] Segal I.: Nonlinear semigroups. Ann. of Math. 78 (1962), 334-362

[26] Taylor A. E.: Introduction to functional analysis, John Wiley and Sons, New York, 1958.

[27] Witthaut D., Mossmann S., Korsch H. J.: Bound and resonance states of the nonlinear Schrödinger equation in simple model systems, J.Phys.A, 38 (2005), 1777-1702 